- Supporting Information -

\title{
Development and profiling of inverse agonist tools for the neuroprotective transcription factor Nurr1
}

Daniel Zaienne ${ }^{1} \ddagger$, Sabine Willems ${ }^{1} \ddagger$, Simone Schierle ${ }^{1}$, Jan Heering ${ }^{2}$, Daniel Merk ${ }^{1,3 *}$

${ }^{1}$ Institute of Pharmaceutical Chemistry, Goethe University Frankfurt, D-60438 Frankfurt, Germany

${ }^{2}$ Fraunhofer Institute for Translational Medicine and Pharmacology ITMP, D-60596 Frankfurt, Germany

${ }^{3}$ Department of Pharmacy, Ludwig-Maximilians-Universität München, D-81377 Munich, Germany

* merk@pharmchem.uni-frankfurt.de

\section{Table of Contents}

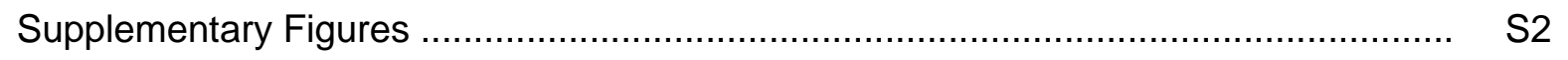

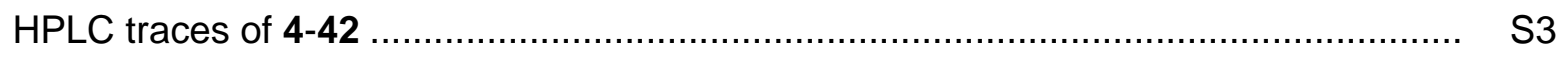




\section{Supplementary Figures}

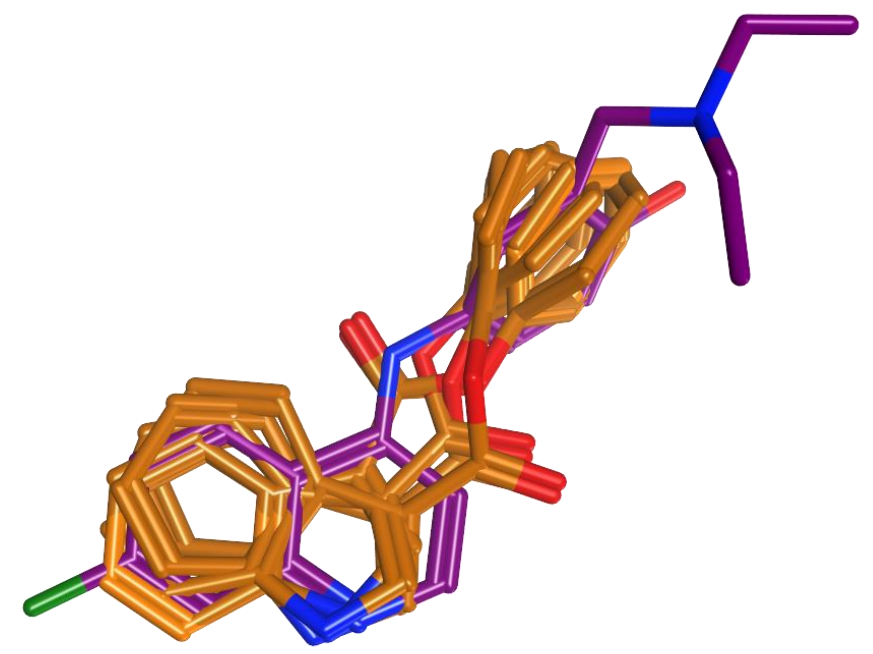

Figure S1. Flexible alignment (MOE) of Nurr1 agonists 25 and $A Q$ reveals structural similarity with superposition of phenyl ester (25) and phenyl amine (AQ). 


\section{HPLC traces of 4-42}

2-Methyl-1H-indole-3-carboxylic acid methyl ester (5):
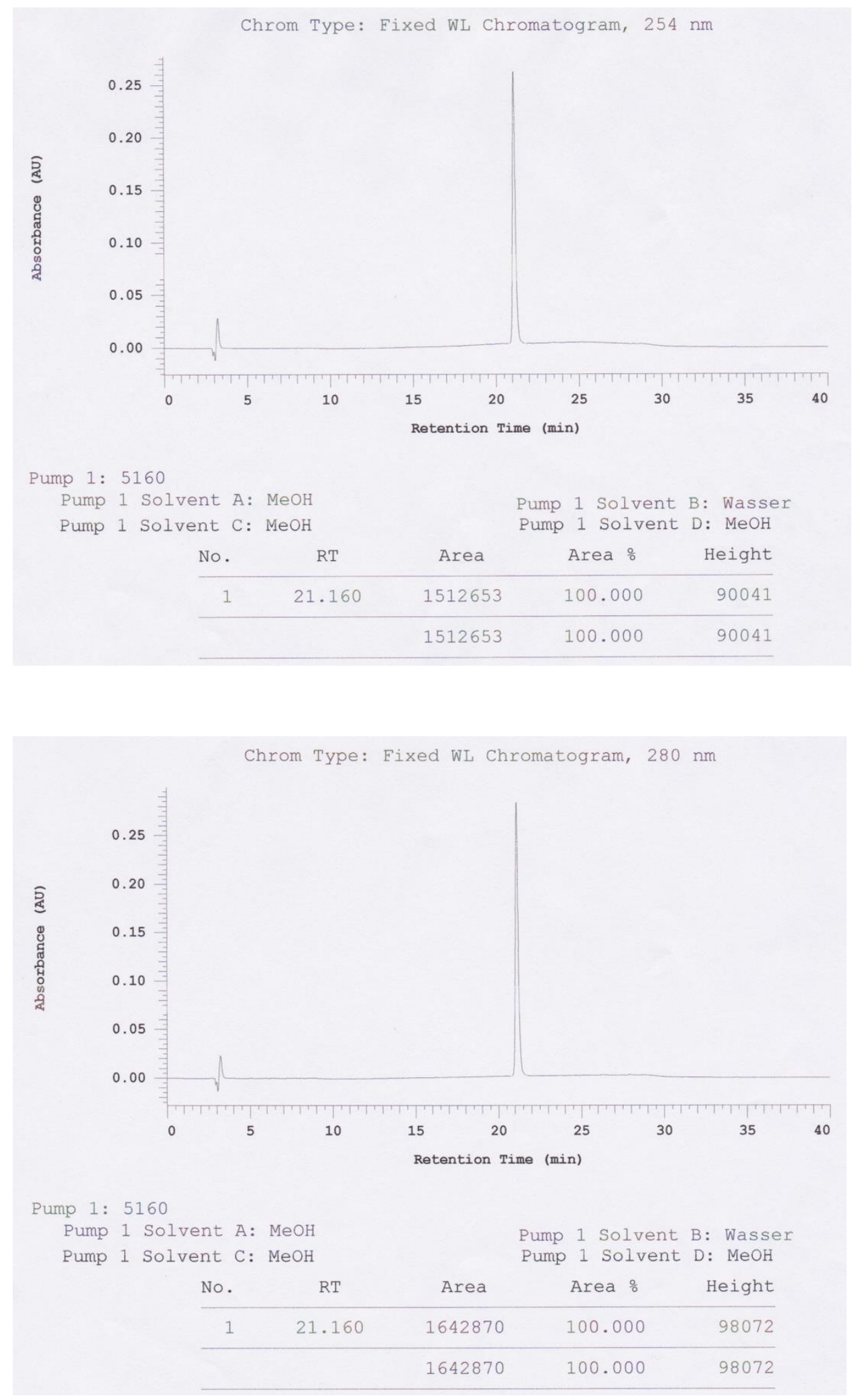


\section{2-Chloro-1H-indole-3-carboxylic acid methyl ester (6):}

Chrom Type: Fixed WL Chromatogram, $254 \mathrm{~nm}$

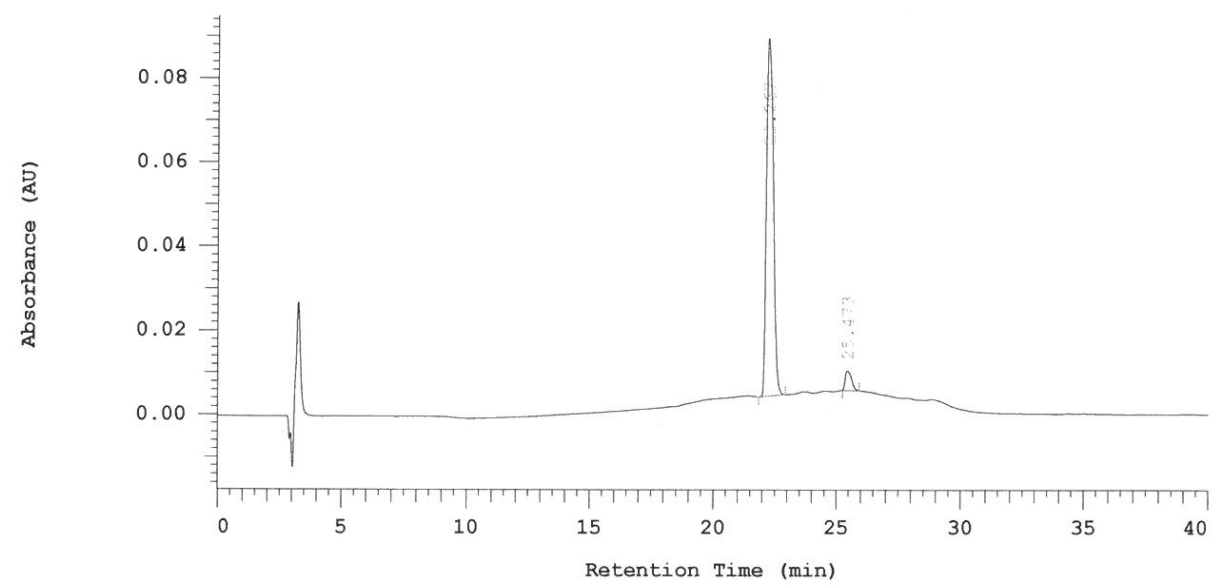

Pump 1: 5160

Pump 1 Solvent A: MeOH Pump 1 Solvent B: Wasser

Pump 1 Solvent C: $\mathrm{MeOH}$

Pump 1 Solvent D: MeOH

\begin{tabular}{rrrrr} 
No. & RT & Area & Area & Height \\
\hline 1 & 22.267 & 810083 & 95.244 & 39340 \\
2 & 25.473 & 40450 & 4.756 & 2249 \\
\hline & & 850533 & 100.000 & 41589 \\
\hline
\end{tabular}

Chrom Type: Fixed WL Chromatogram, $280 \mathrm{~nm}$

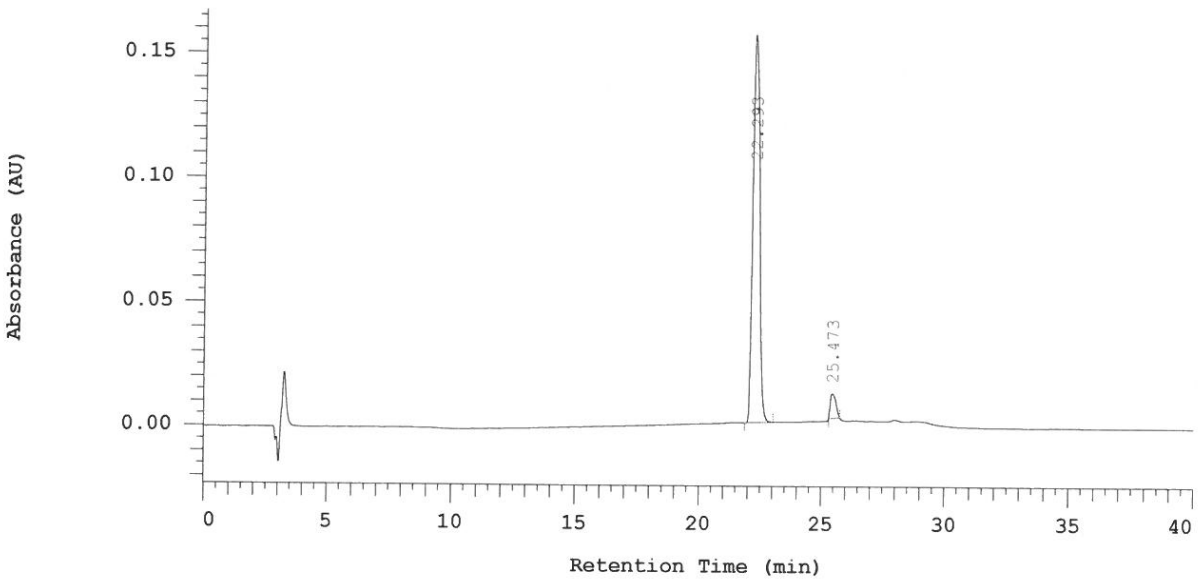

Pump 1: 5160

Pump 1 Solvent A: $\mathrm{MeOH}$

Pump 1 Solvent C: $\mathrm{MeOH}$

Pump 1 Solvent B: Wasser

Pump 1 Solvent D: $\mathrm{MeOH}$

\begin{tabular}{rrrrr} 
No. & RT & Area & Area \% & Height \\
\hline 1 & 22.293 & 1489299 & 95.109 & 68869 \\
2 & 25.473 & 76591 & 4.891 & 4740 \\
\hline & & 1565890 & 100.000 & 73609 \\
\hline
\end{tabular}


4-Methyl-1H-indole-3-carboxylic acid methyl ester (7):
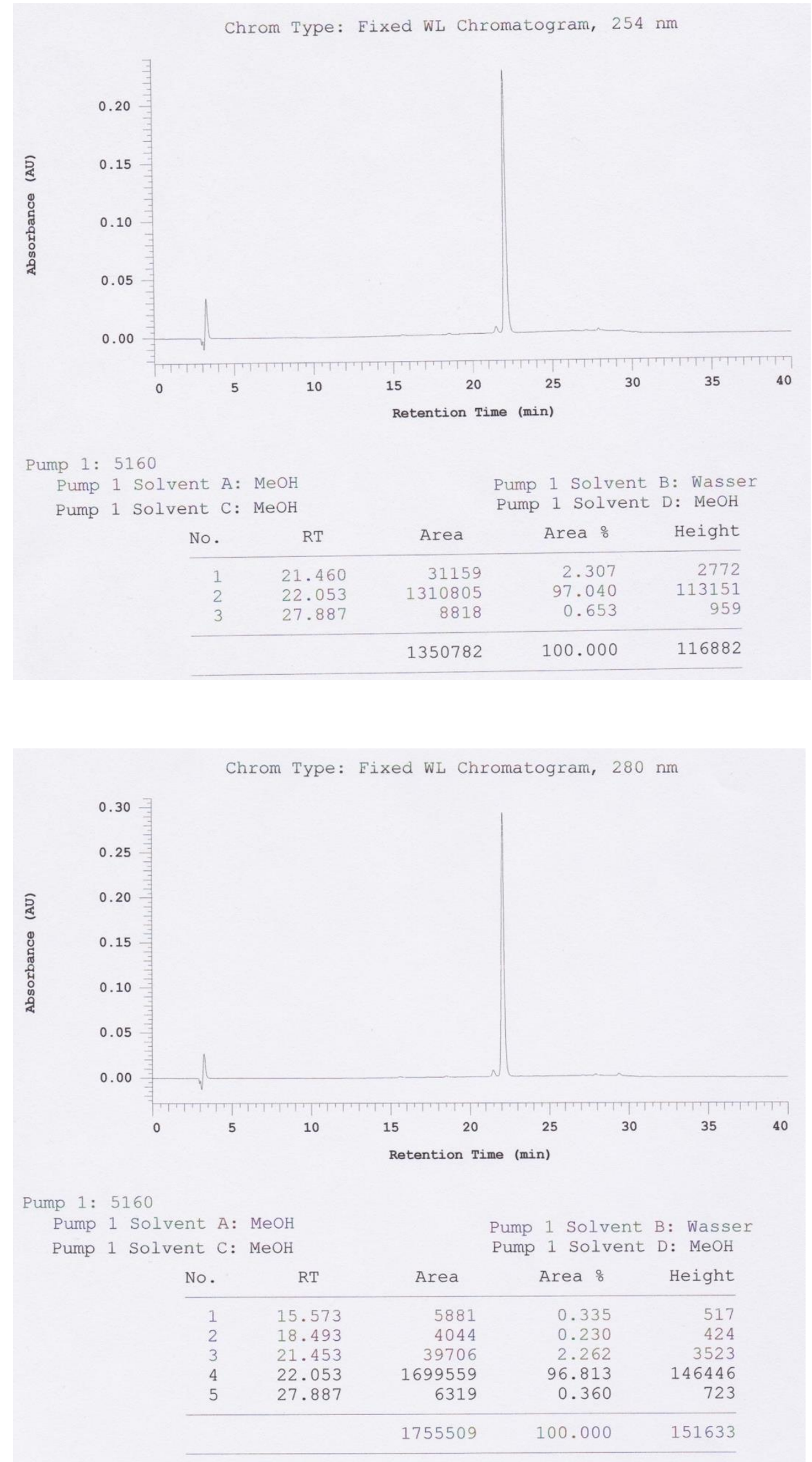
4-Chloro-1H-indole-3-carboxylic acid methyl ester (8):
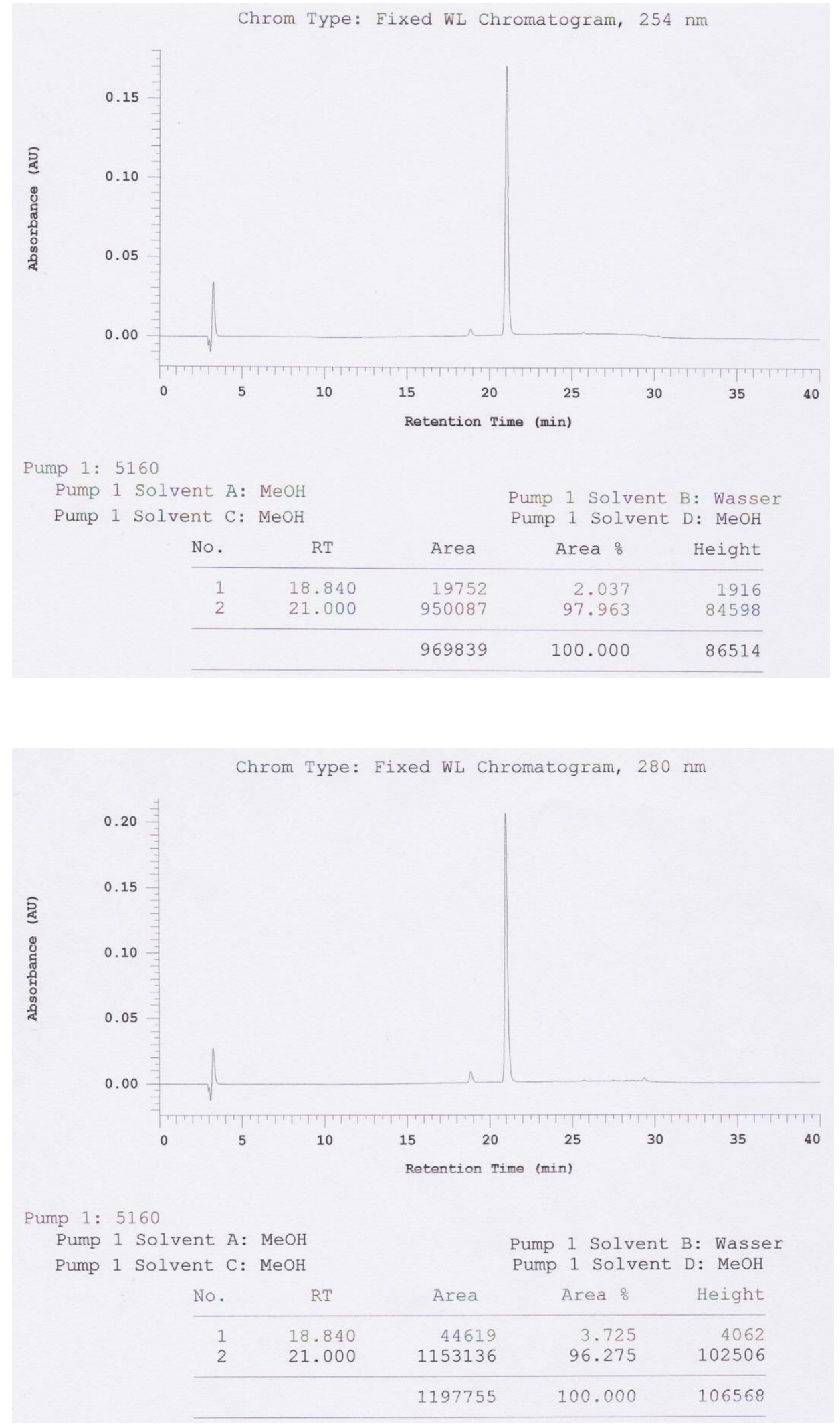
5-Methyl-1H-indole-3-carboxylic acid methyl ester (9):
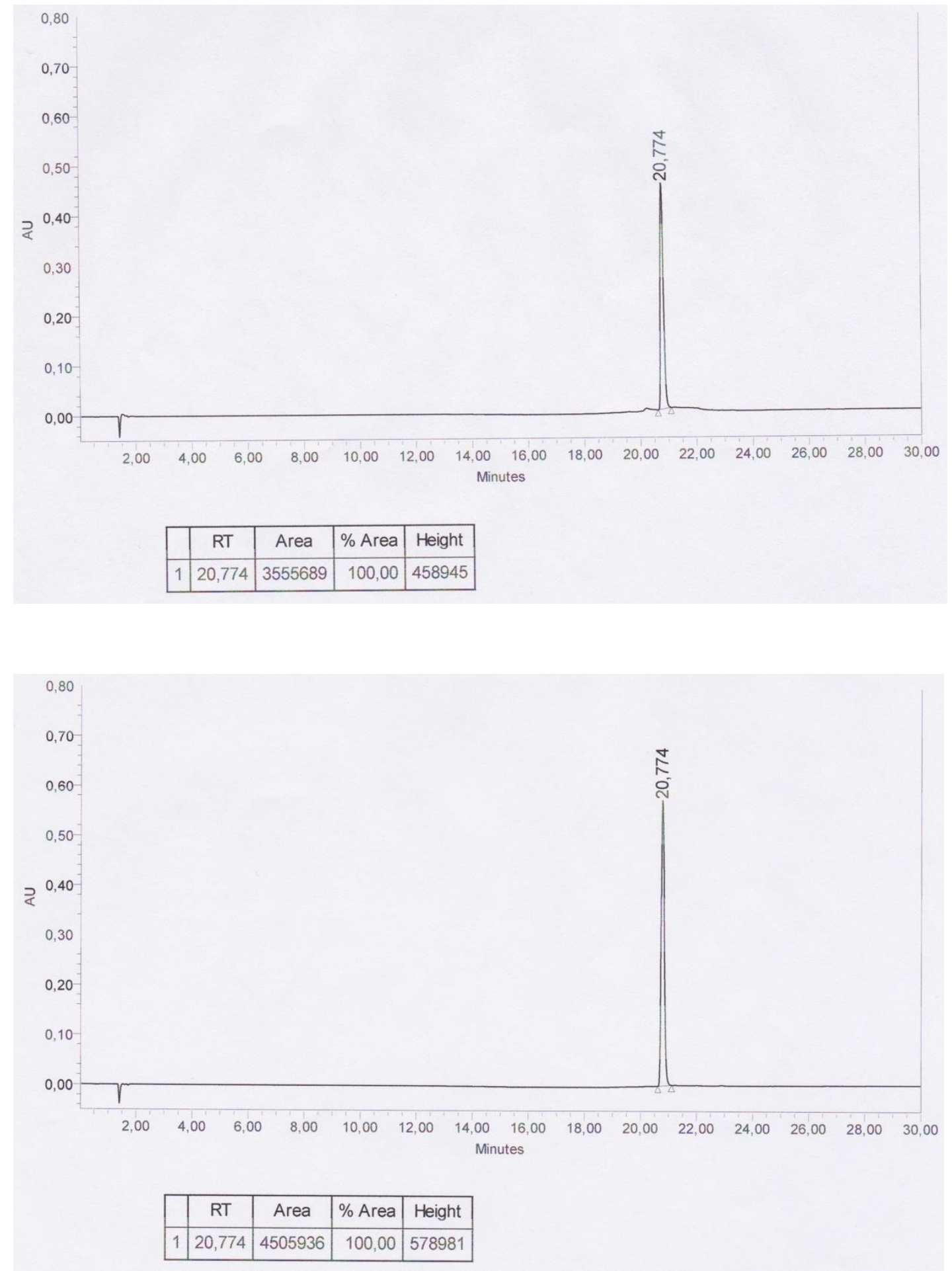
5-Chloro-1 $\mathrm{H}$-indole-3-carboxylic acid methyl ester (10):

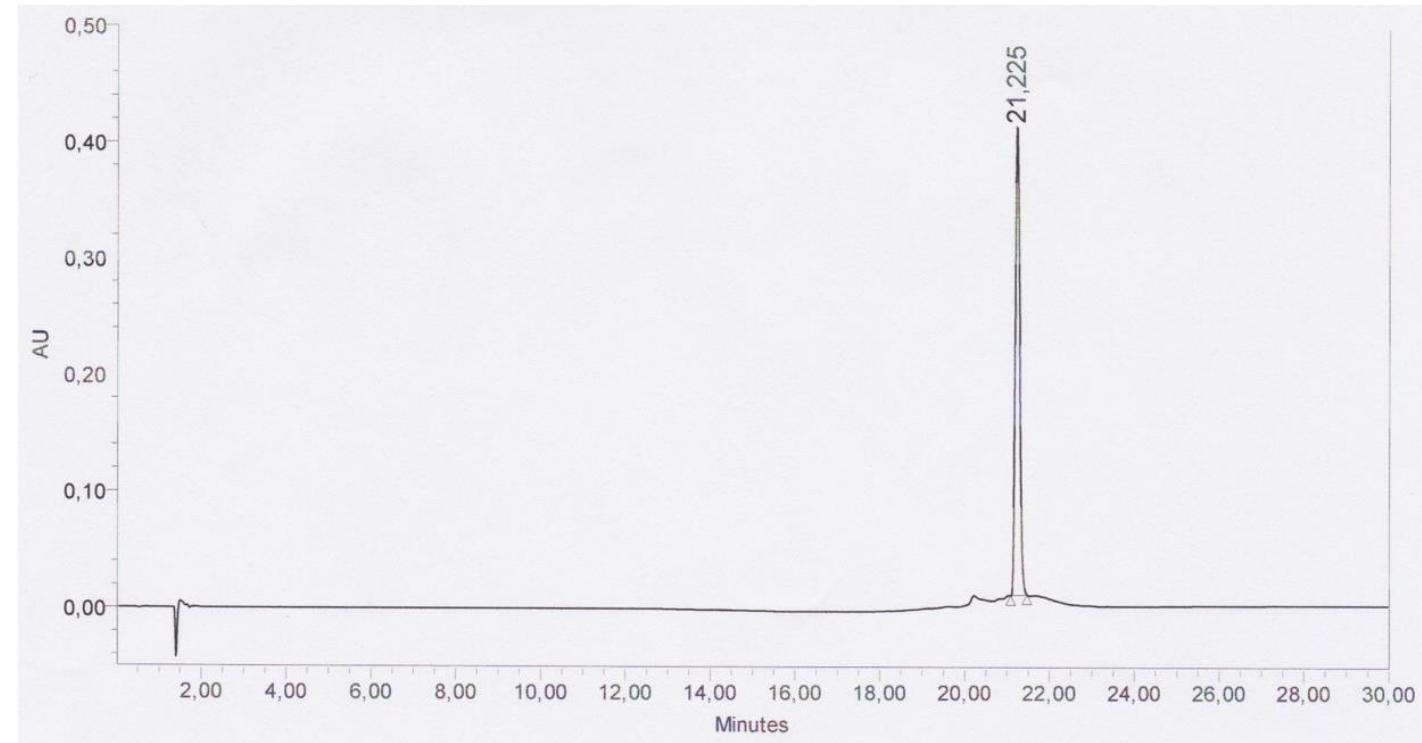

\begin{tabular}{|c|c|c|c|c|}
\hline & RT & Area & $\%$ Area & Height \\
\hline 1 & 21,225 & 2992706 & 100,00 & 407965 \\
\hline
\end{tabular}

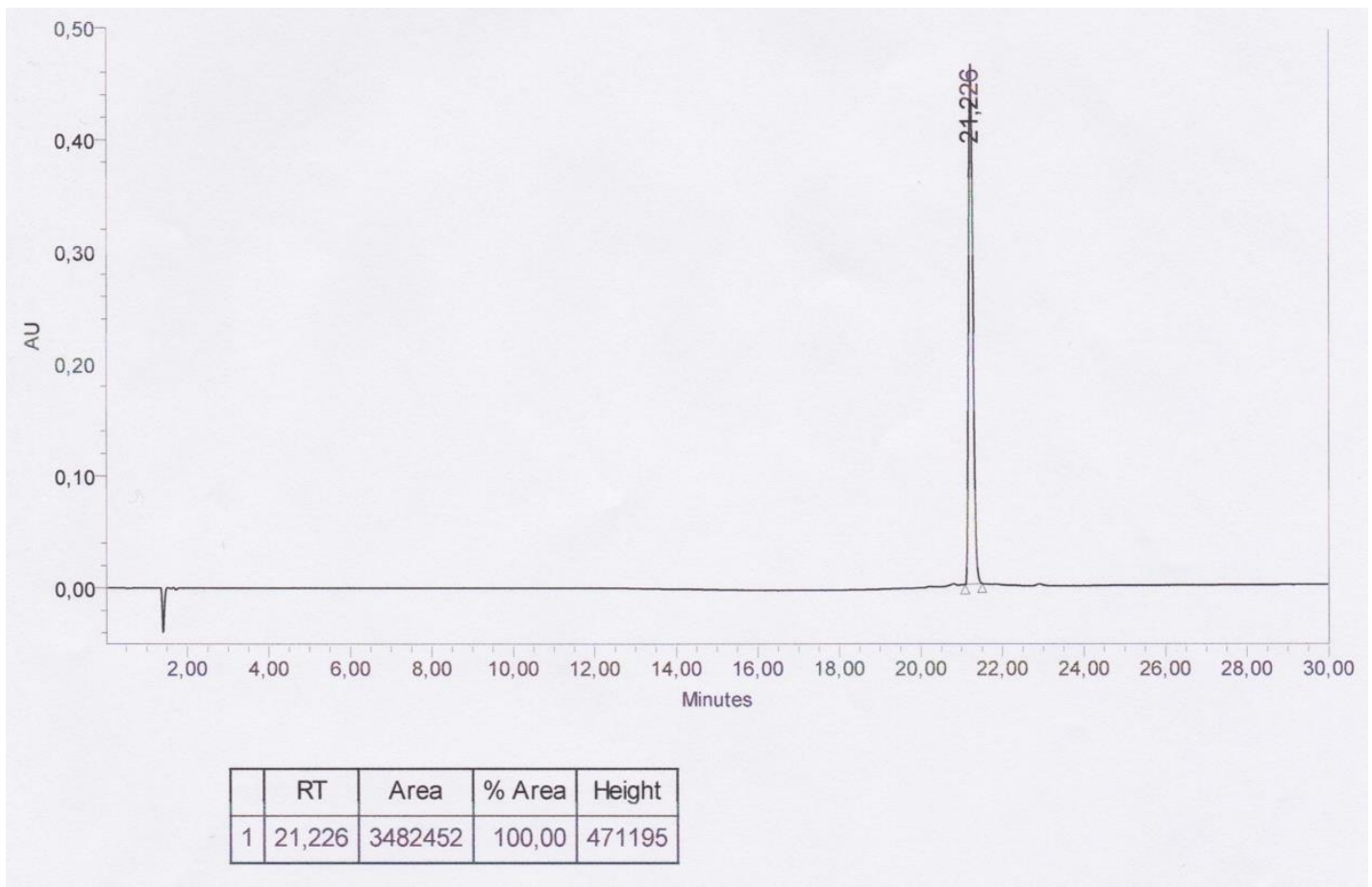


6-Methyl-1H-indole-3-carboxylic acid methyl ester (11):
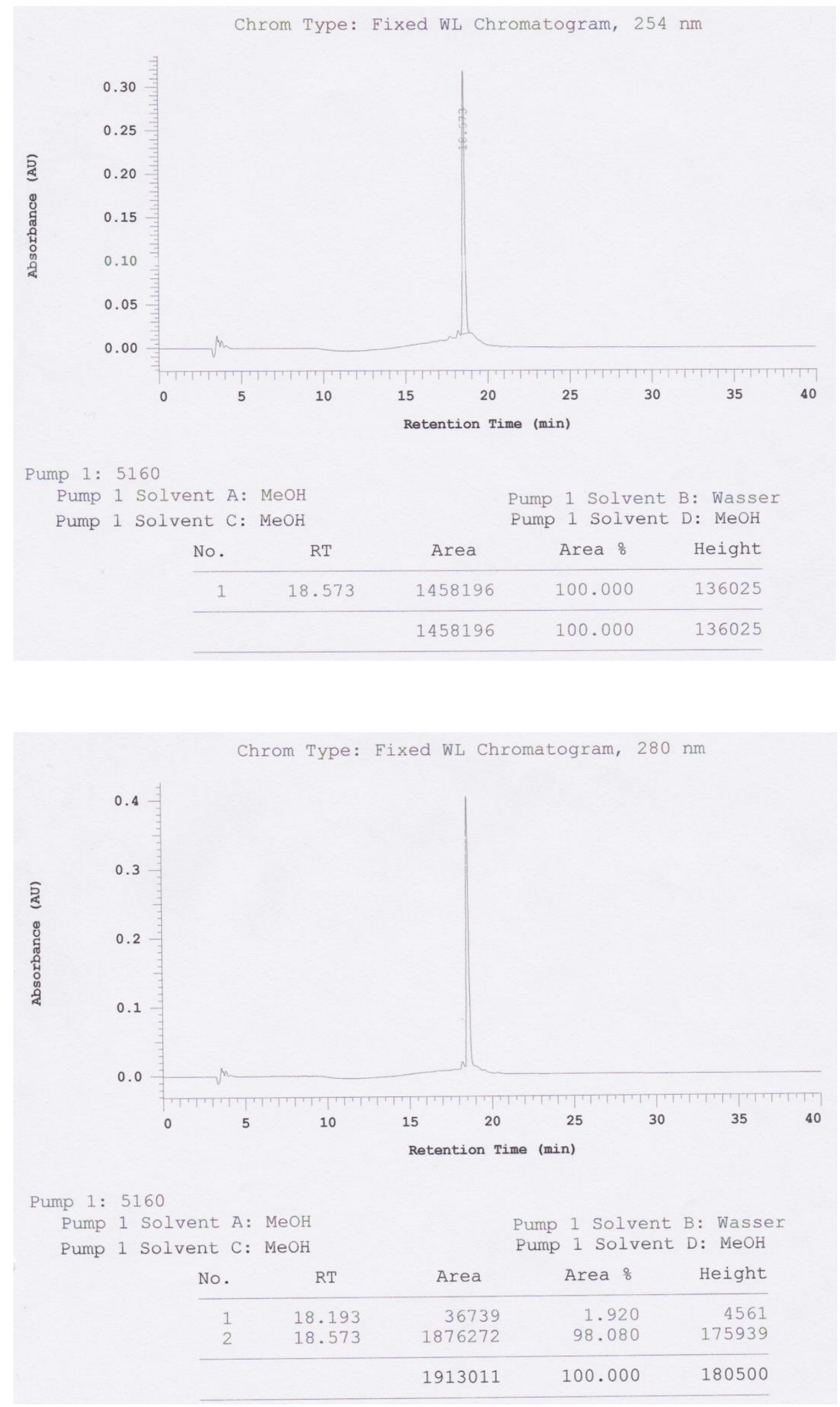
6-Chloro-1H-indole-3-carboxylic acid methyl ester (12):
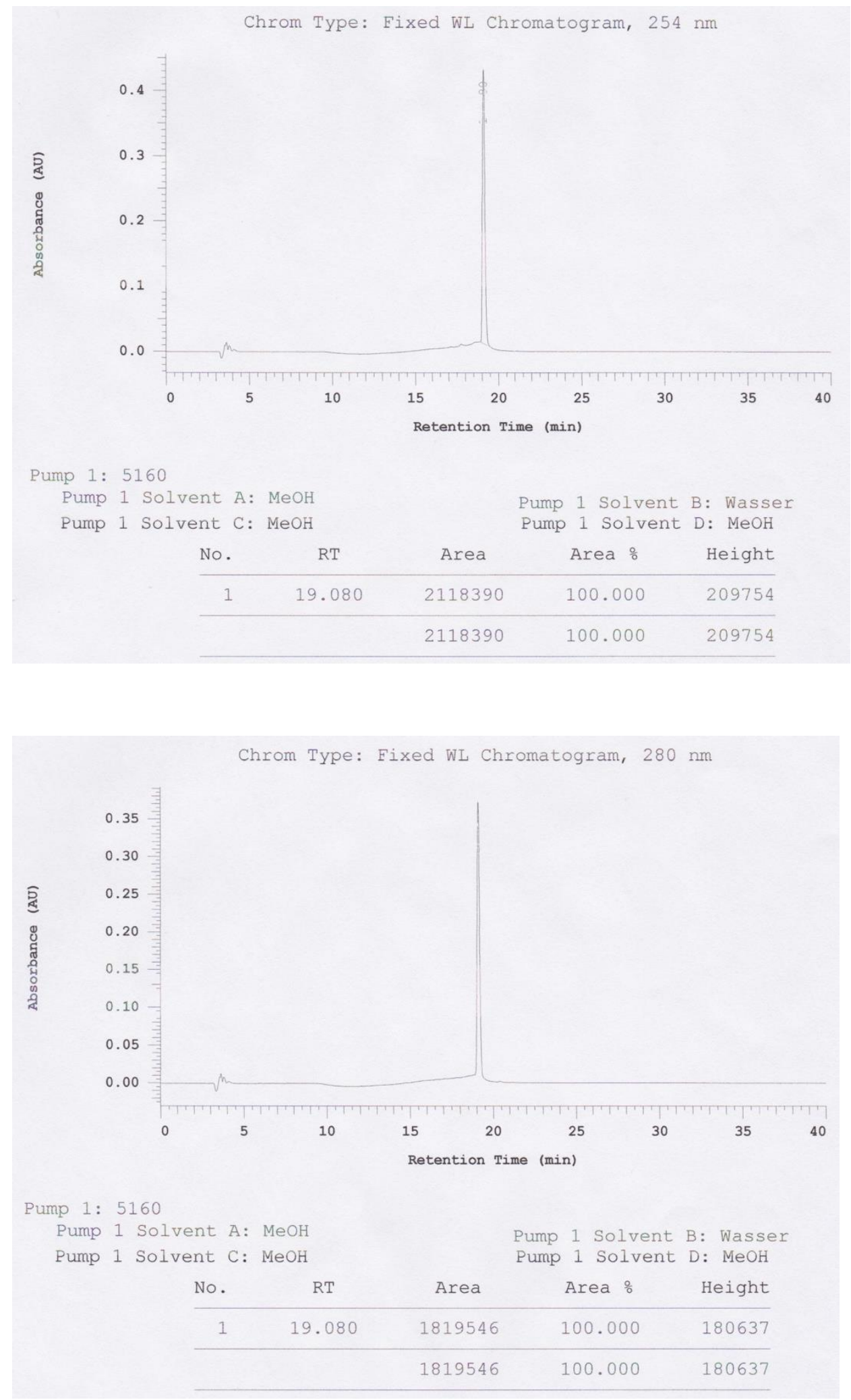
7-Methyl-1H-indole-3-carboxylic acid methyl ester (13):
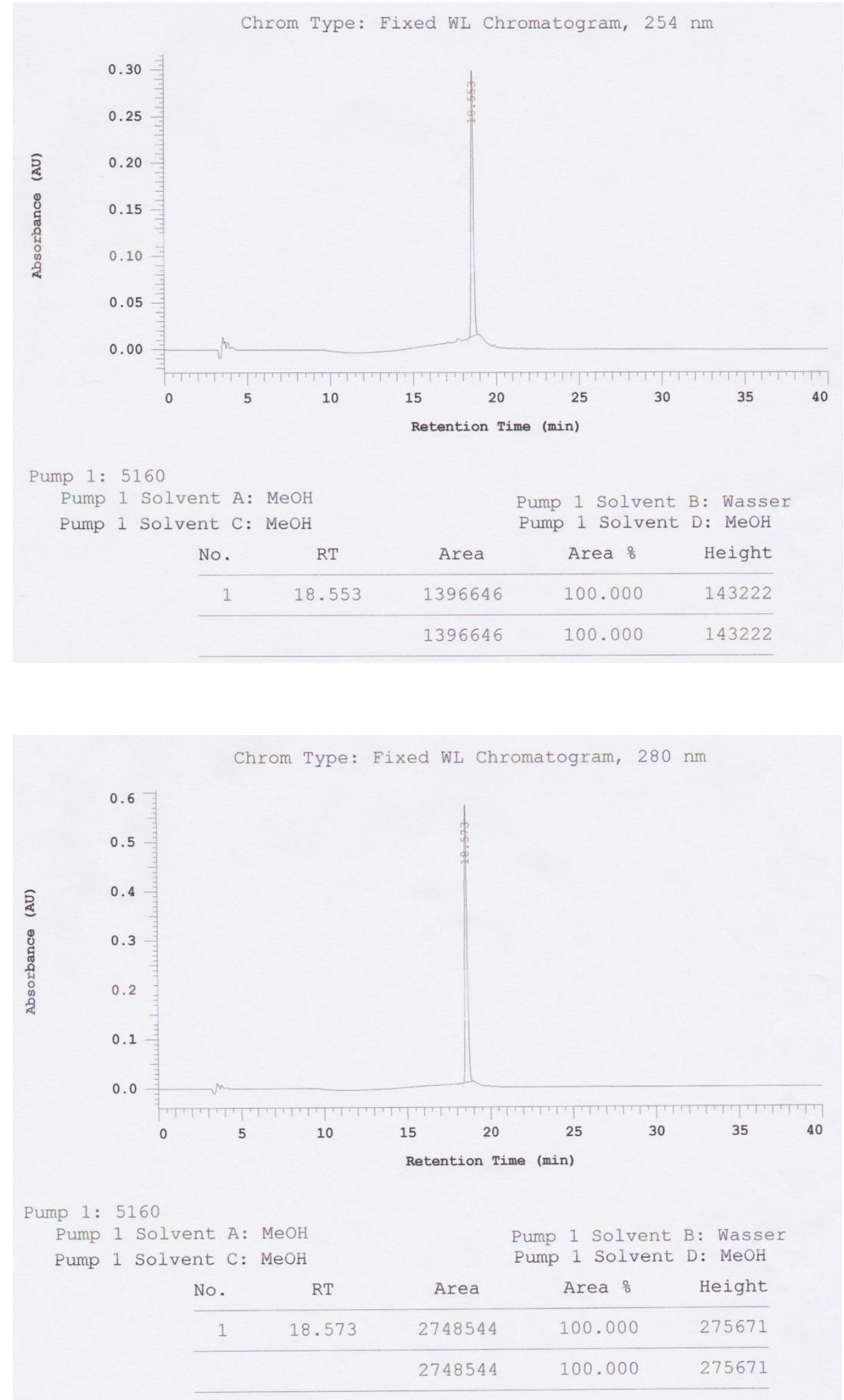
7-Chloro-1 $\mathrm{H}$-indole-3-carboxylic acid methyl ester (14):
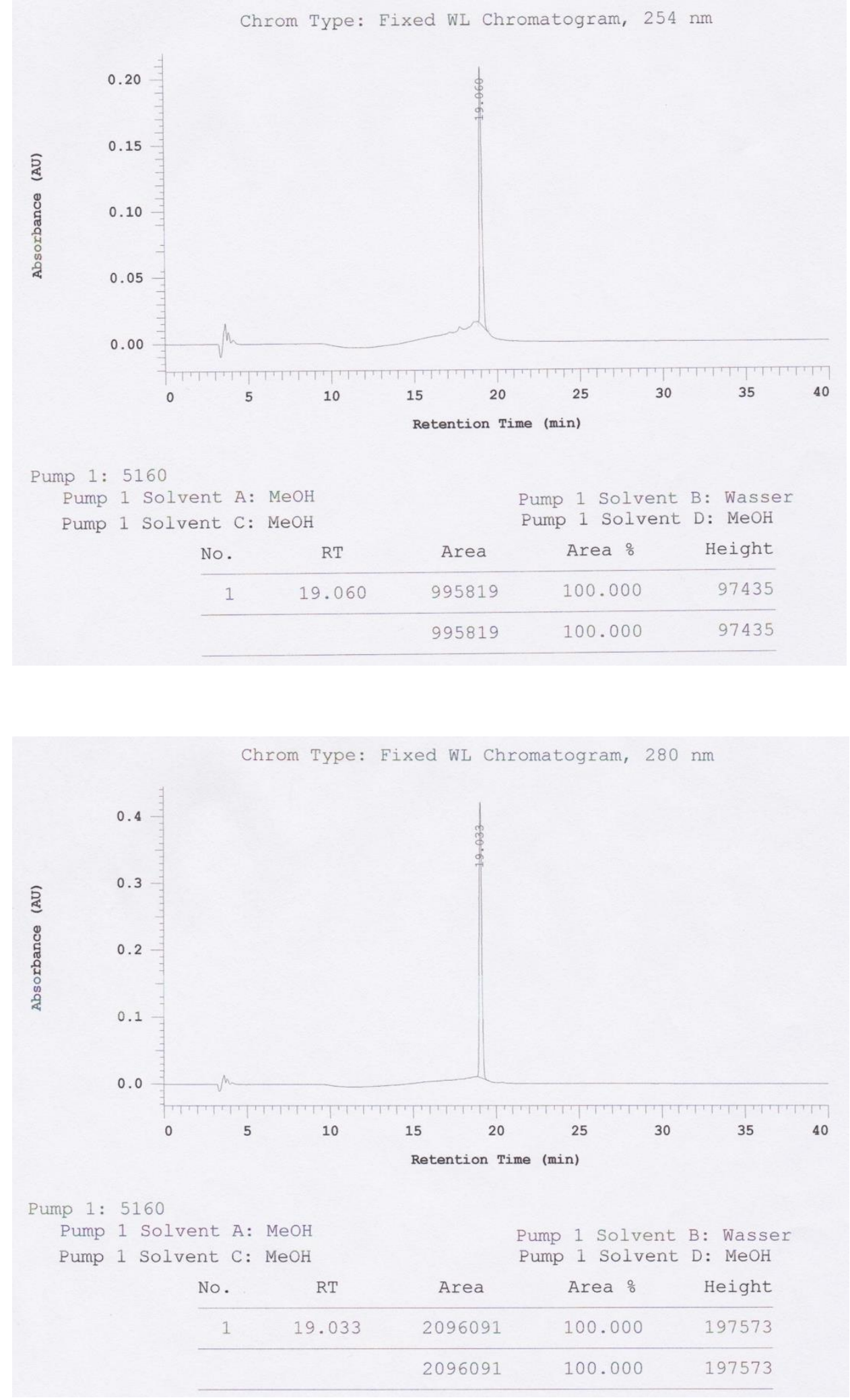
1-Methylindole-3-carboxylic acid methyl ester (15):
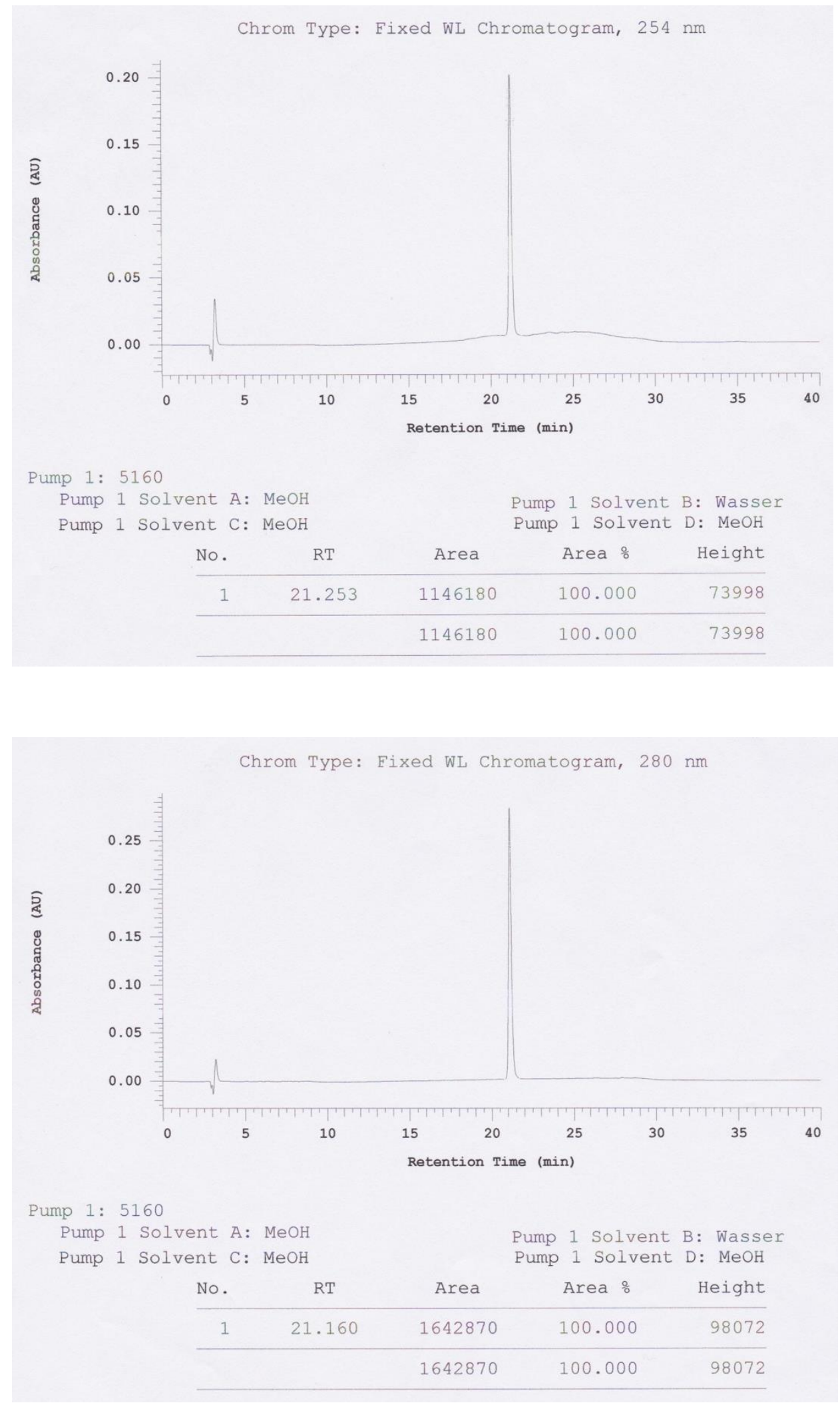


\section{1-Ethylindole-3-carboxylic acid methyl ester (16):}
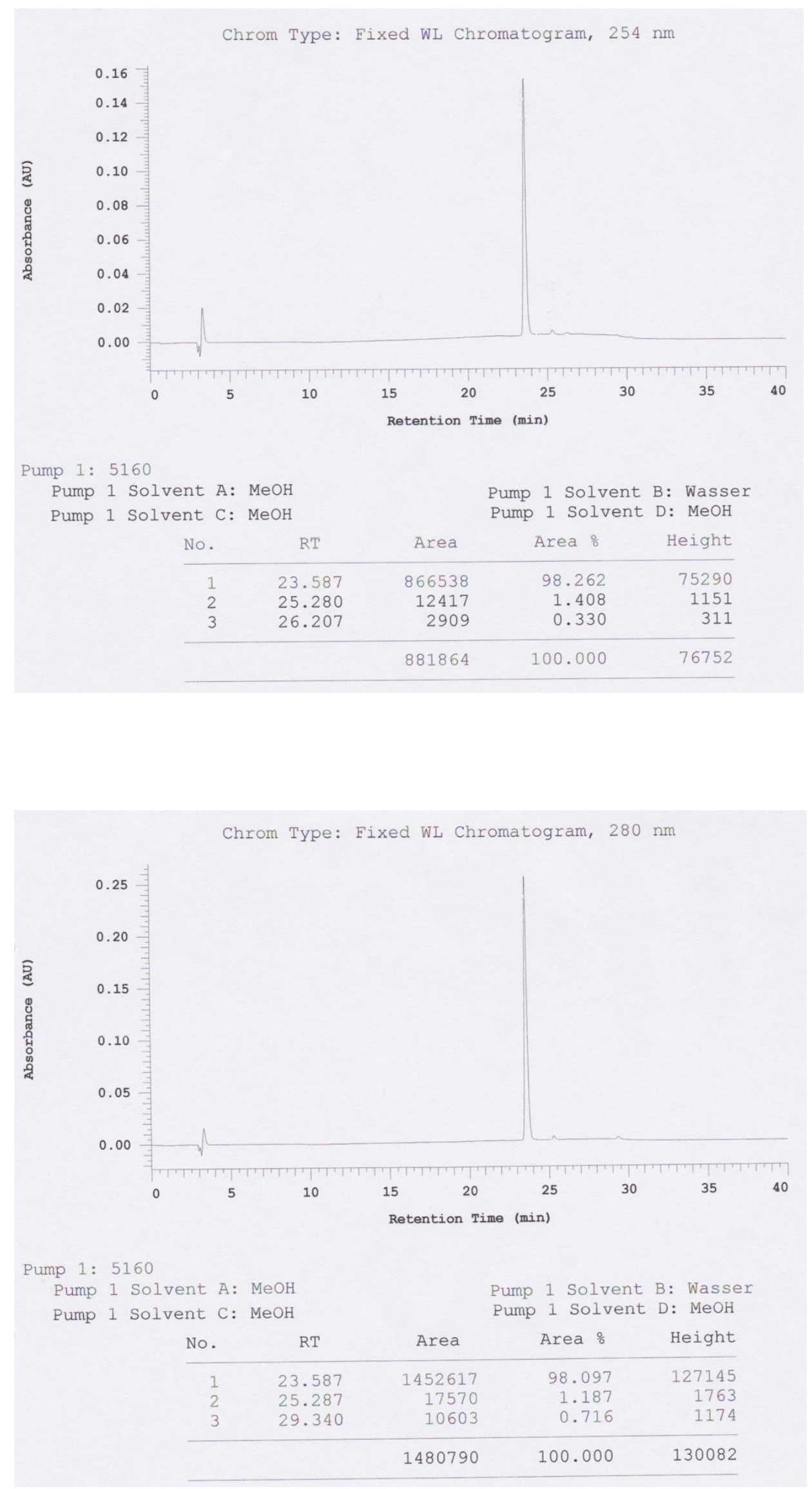

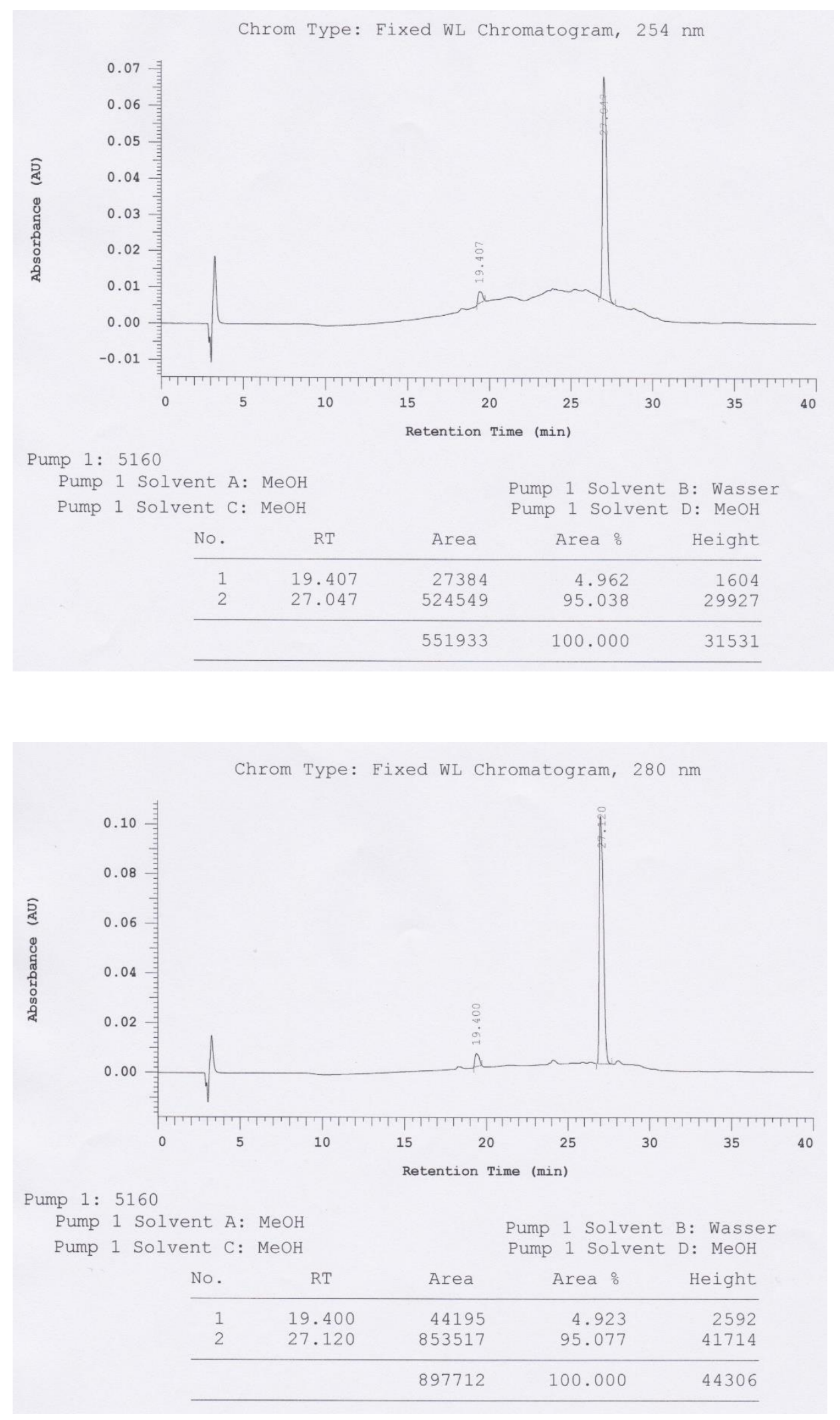
1-Benzylindole-3-carboxylic acid methyl ester (18):
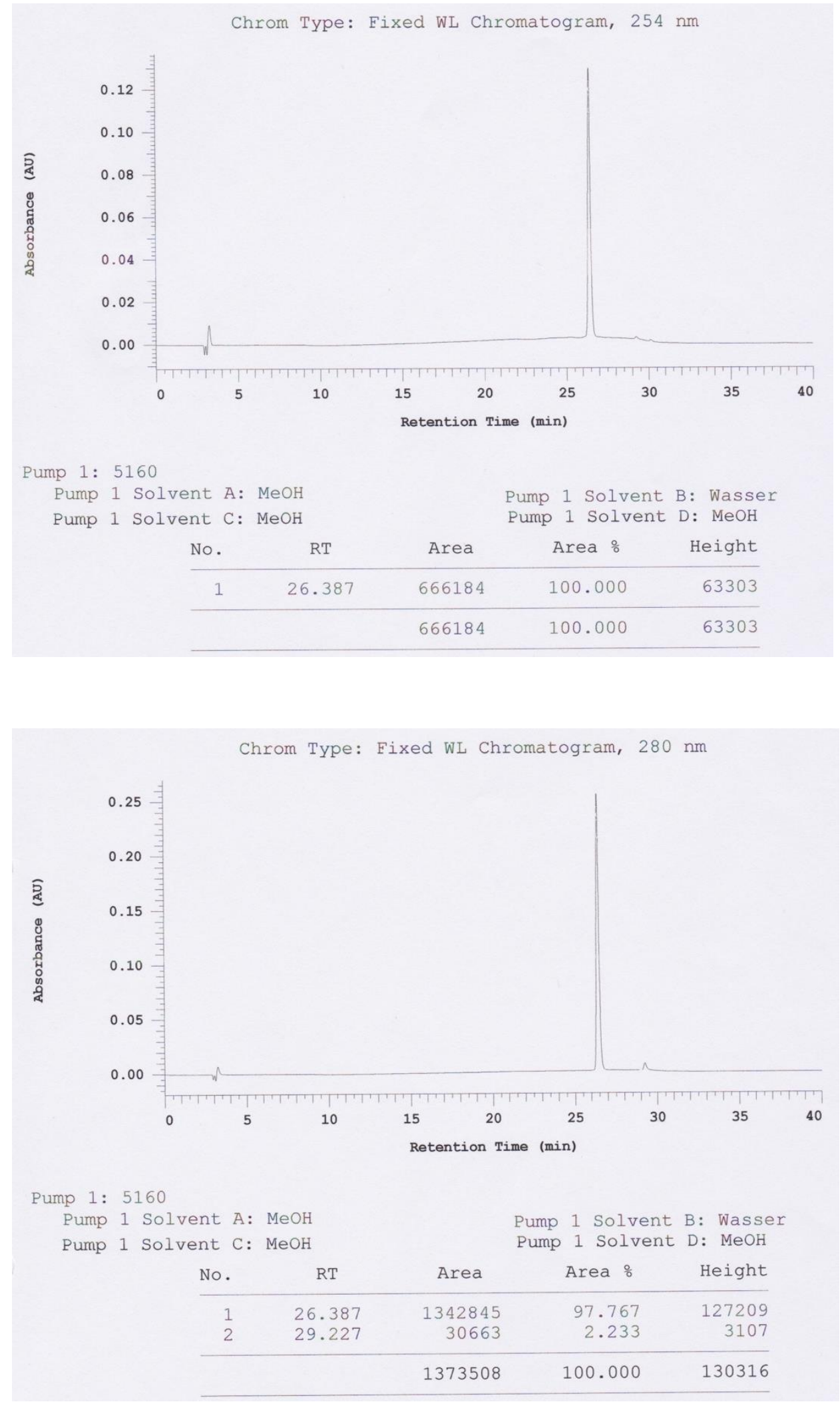
1H-Indole-3-carboxylic acid (19):

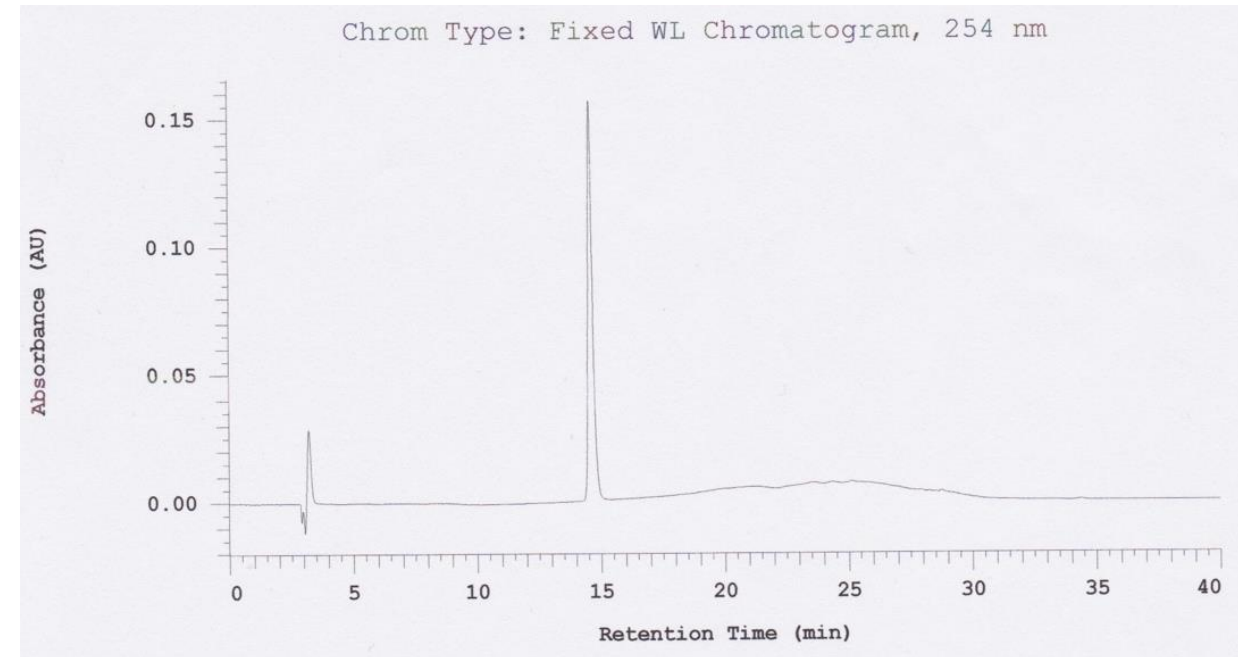

Pump 1: 5160

Pump 1 Solvent A: MeOH Pump 1 Solvent B: Wasser

Pump 1 Solvent C: $\mathrm{MeOH}$

Pump 1 Solvent D: $\mathrm{MeOH}$

\begin{tabular}{ccccc} 
No. & RT & Area & Area \% & Height \\
\hline 1 & 14.667 & 1011775 & 100.000 & 53224 \\
\hline & 1011775 & 100.000 & 53224
\end{tabular}

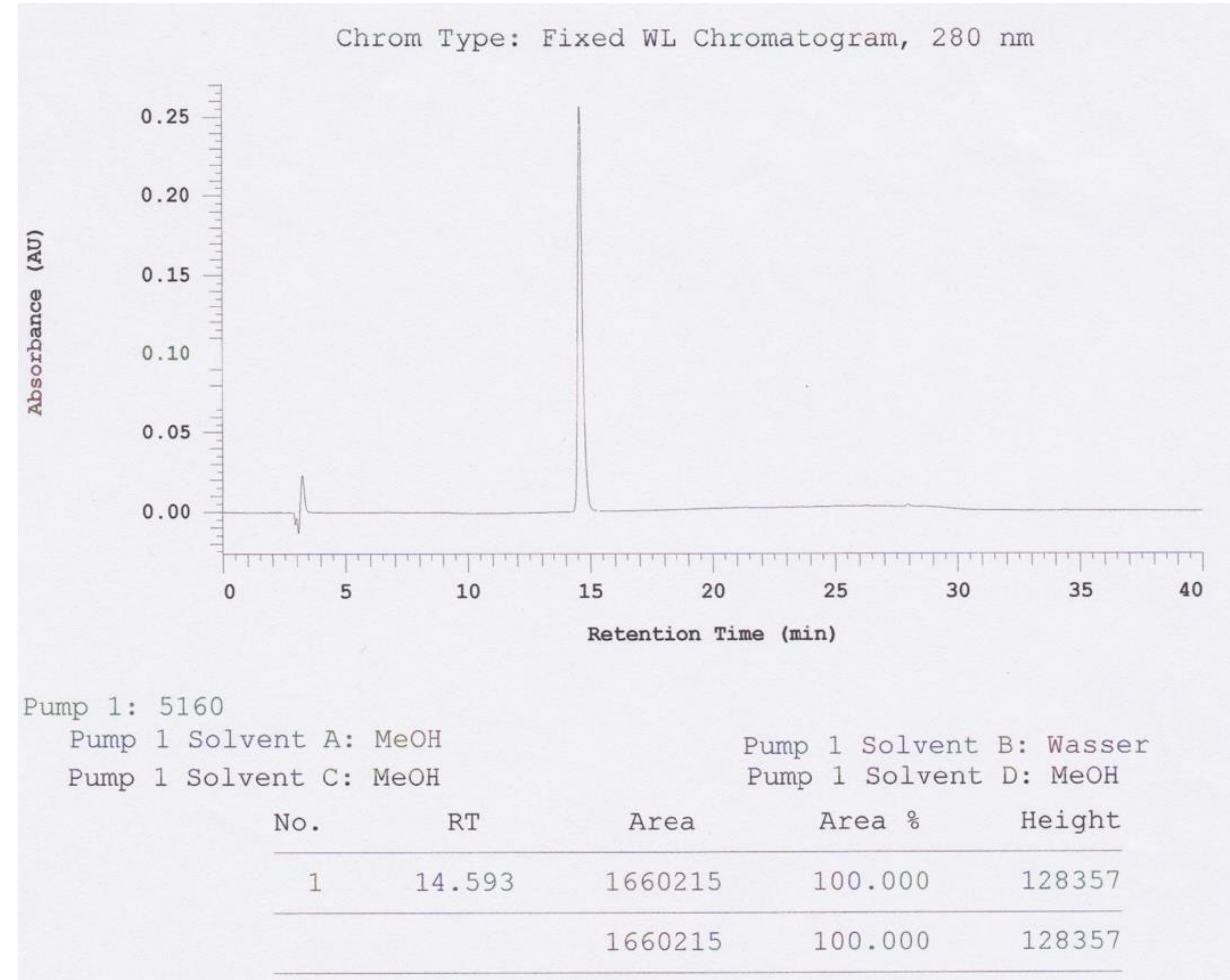


$1 H$-Indole-3-propan-1-one (22):

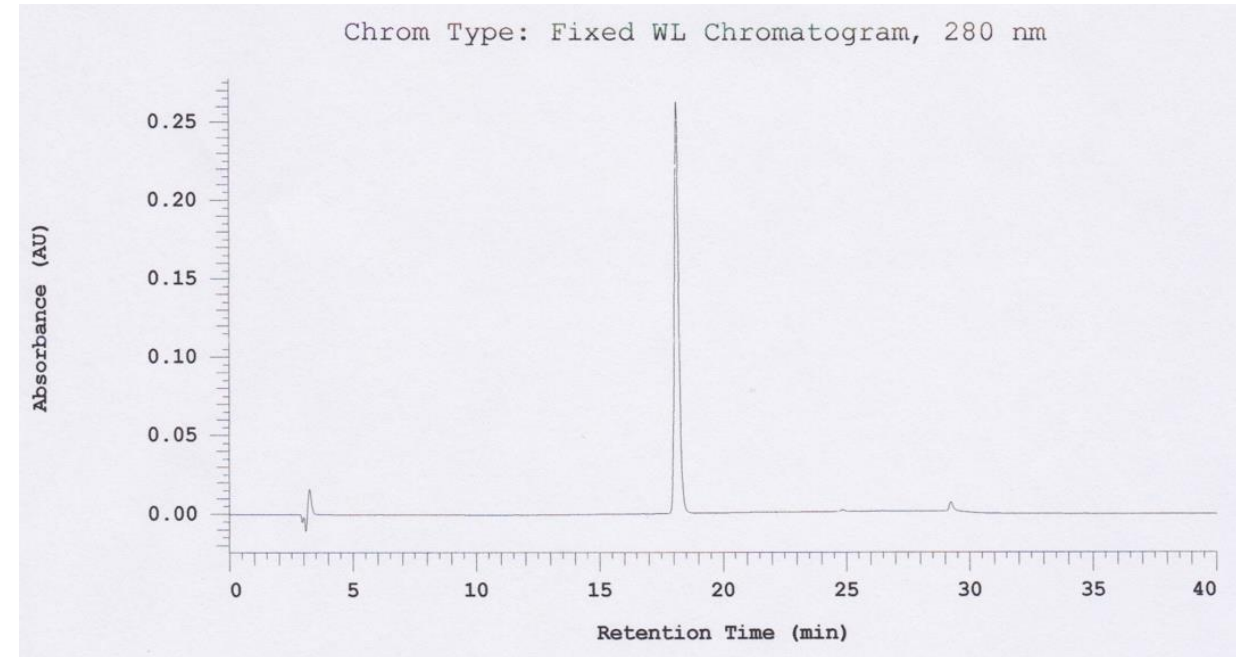

Pump 1: 5160

Pump 1 Solvent A: $\mathrm{MeOH} \quad$ Pump 1 Solvent B: Wasser

Pump 1 Solvent C: $\mathrm{MeOH}$ Pump 1 Solvent D: MeOH

\begin{tabular}{rrrrr} 
No. & \multicolumn{1}{c}{ RT } & \multicolumn{1}{c}{ Area } & Area & Height \\
\hline 1 & 18.113 & 1575360 & 98.019 & 131225 \\
2 & 24.807 & 5873 & 0.365 & 558 \\
3 & 29.220 & 25966 & 1.616 & 2739 \\
\hline & & 1607199 & 100.000 & 134522 \\
\hline
\end{tabular}

Chrom Type: Fixed WL Chromatogram, 254 nm

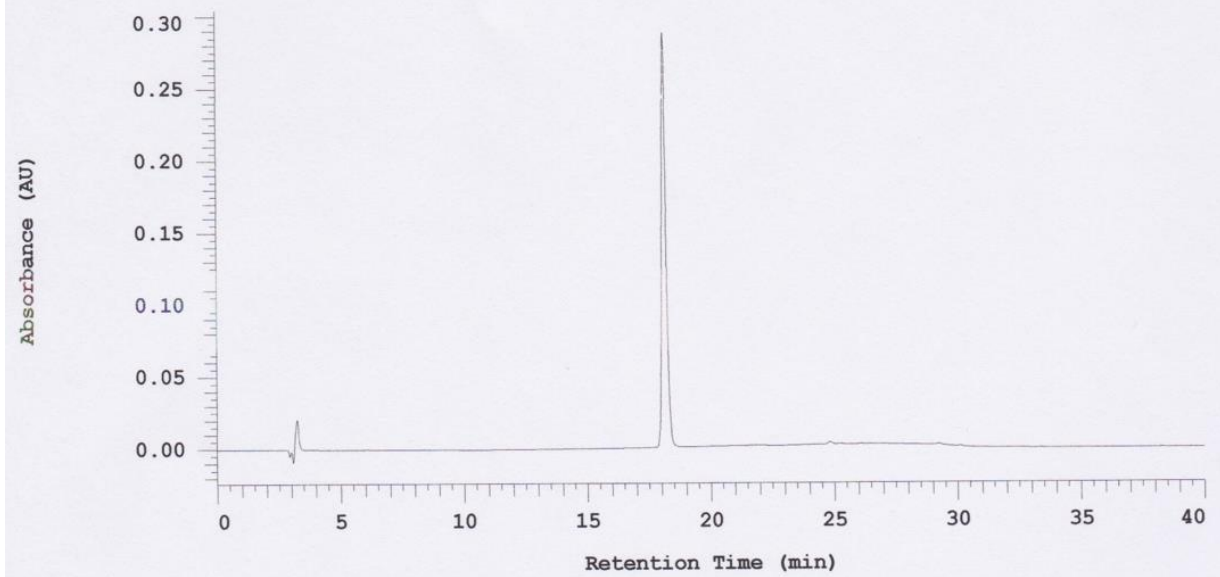

Pump 1: 5160

Pump 1 Solvent A: $\mathrm{MeOH}$

Pump 1 Solvent B: Wasser

Pump 1 Solvent C: $\mathrm{MeOH}$

Pump 1 Solvent D: $\mathrm{MeOH}$

\begin{tabular}{ccccc} 
No. & RT & Area & Area \% & Height \\
\hline 1 & 18.113 & 1730283 & 100.000 & 143960 \\
\hline & 1730283 & 100.000 & 143960 \\
\hline
\end{tabular}


$1 H$-Indole-3-carboxylic acid ethyl ester (23):
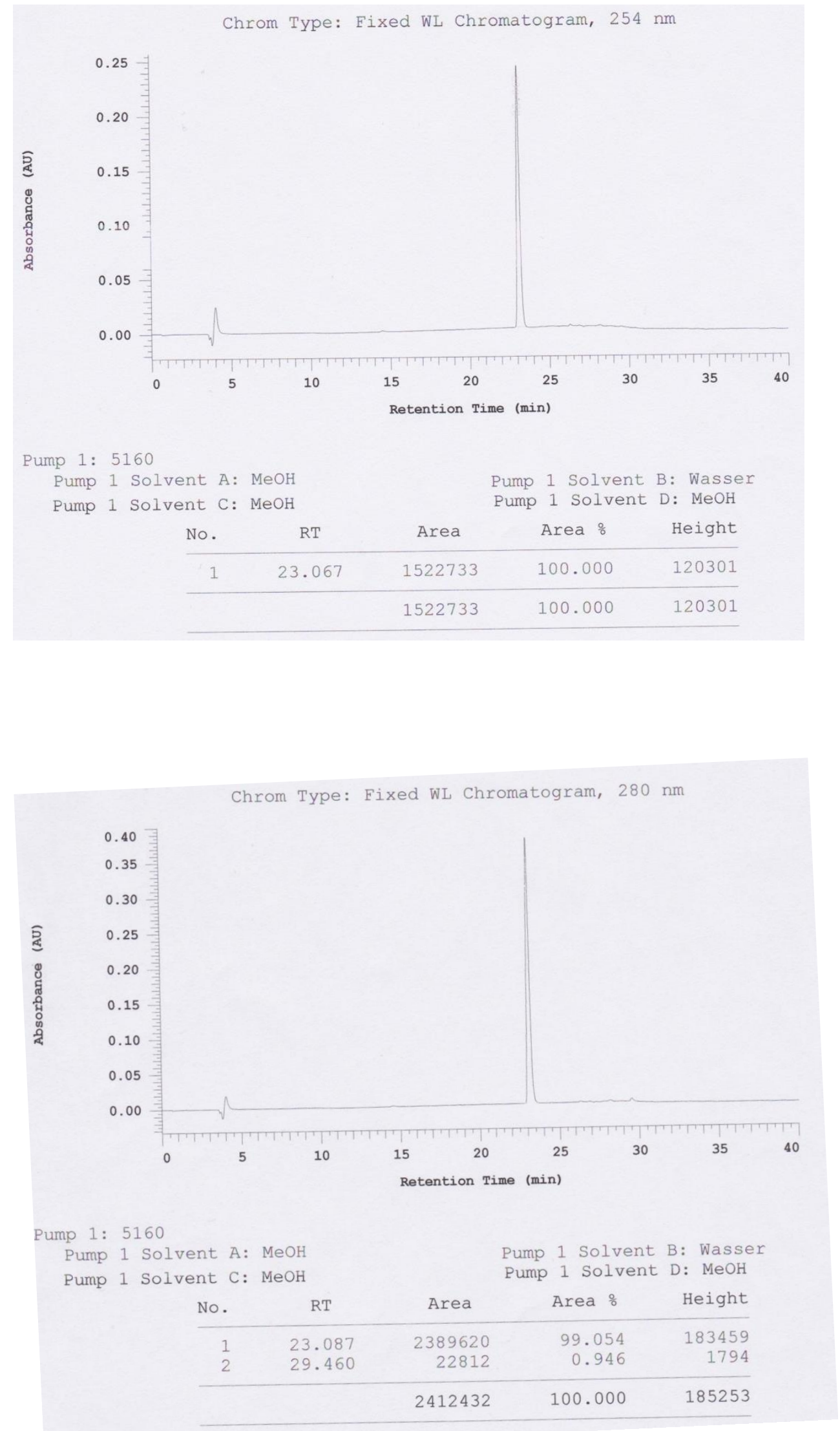
$1 H$-Indole-3-carboxylic tert butyl ester (24):
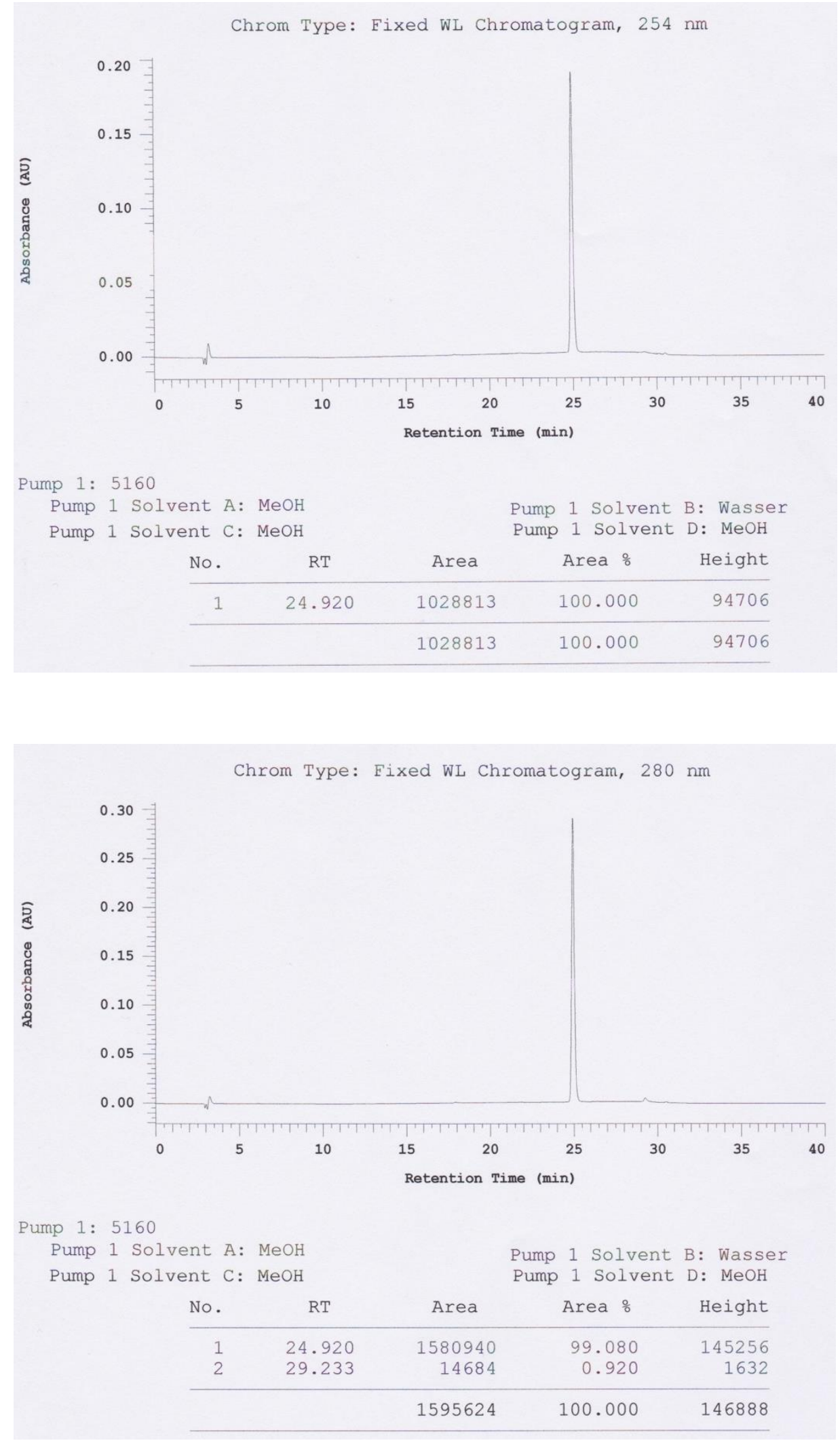
$1 H$-Indole-3 carboxylic acid phenyl ester (25):

Chrom Type: Eixed WL Chromatogram, 254 nm

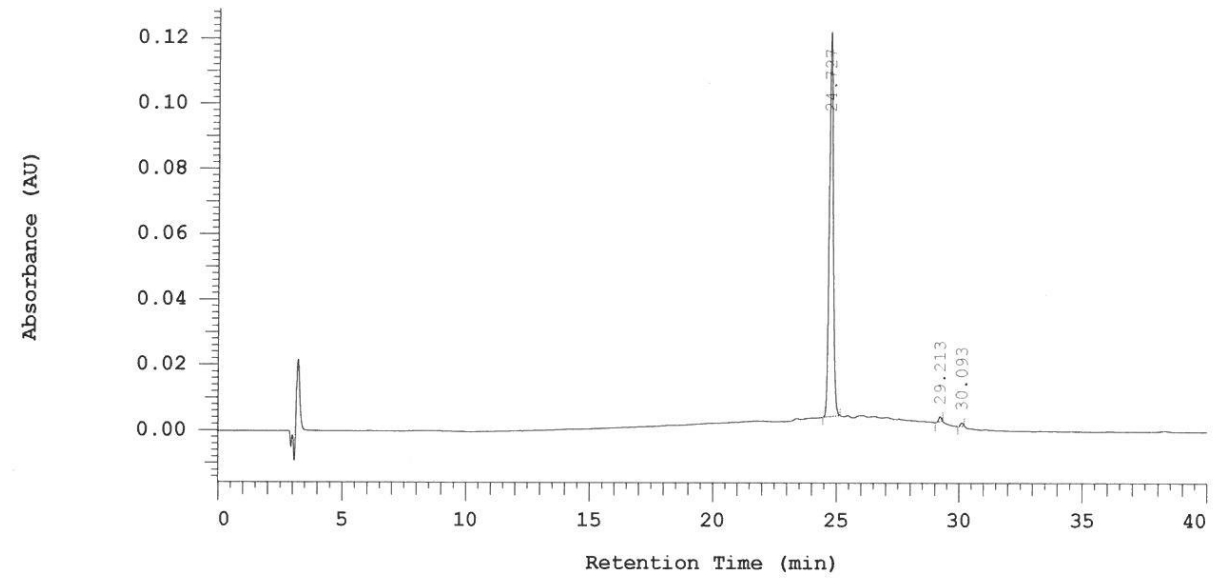

Pump 1: 5160

Pump 1 Solvent A: $\mathrm{MeOH} \quad$ Pump 1 Solvent B: Wasser

Pump 1 Solvent $\mathrm{C}: \mathrm{MeOH} \quad$ Pump 1 Solvent D: MeOH

\begin{tabular}{rrrrr} 
No. & \multicolumn{1}{c}{ RT } & \multicolumn{1}{c}{ Area } & Area & Height \\
\hline 1 & 24.727 & 633365 & 98.620 & 58842 \\
2 & 29.213 & 5053 & 0.787 & 701 \\
3 & 30.093 & 3812 & 0.594 & 499 \\
\hline & 642230 & 100.000 & 60042
\end{tabular}

Chrom Type: Fixed WL Chromatogram, $280 \mathrm{~nm}$

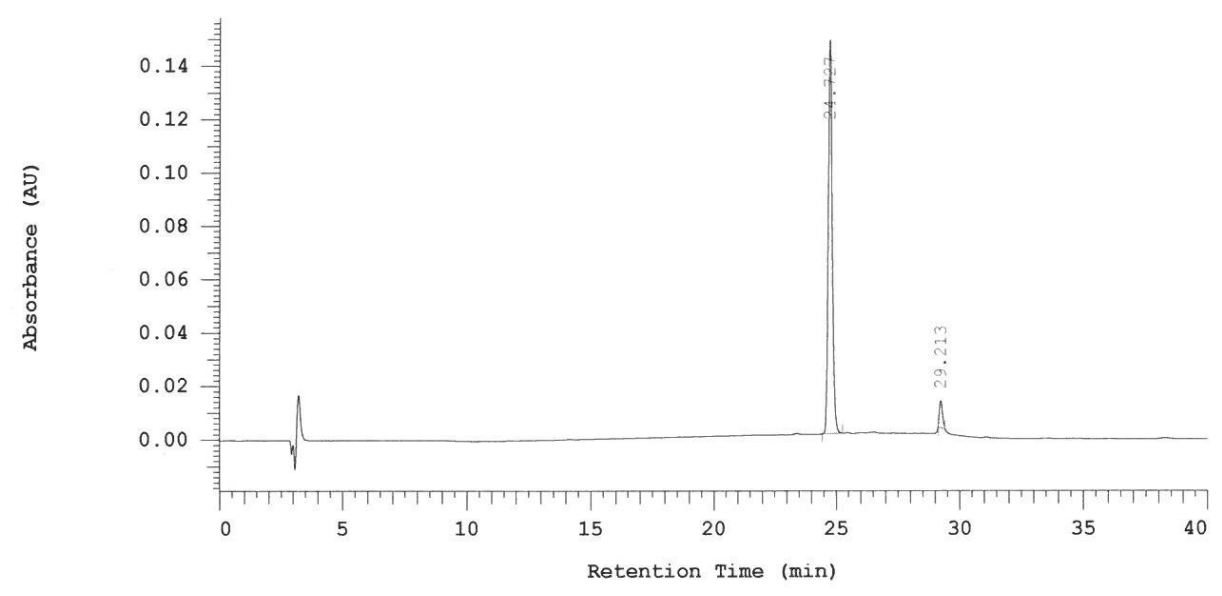

Pump 1: 5160

Pump 1 Solvent A: $\mathrm{MeOH}$

Pump 1 Solvent $\mathrm{C}: \mathrm{MeOH}$

Pump 1 Solvent B: Wasser

Pump 1 Solvent D: $\mathrm{MeOH}$

\begin{tabular}{rrrrr} 
No. & RT & Area & Area & Height \\
\hline 1 & 24.727 & 796102 & 95.008 & 73749 \\
2 & 29.213 & 41828 & 4.992 & 4985 \\
\hline & & 837930 & 100.000 & 78734
\end{tabular}




\section{5-Methoxy-1H-indole-3-carboxylic acid methyl ester (26):}
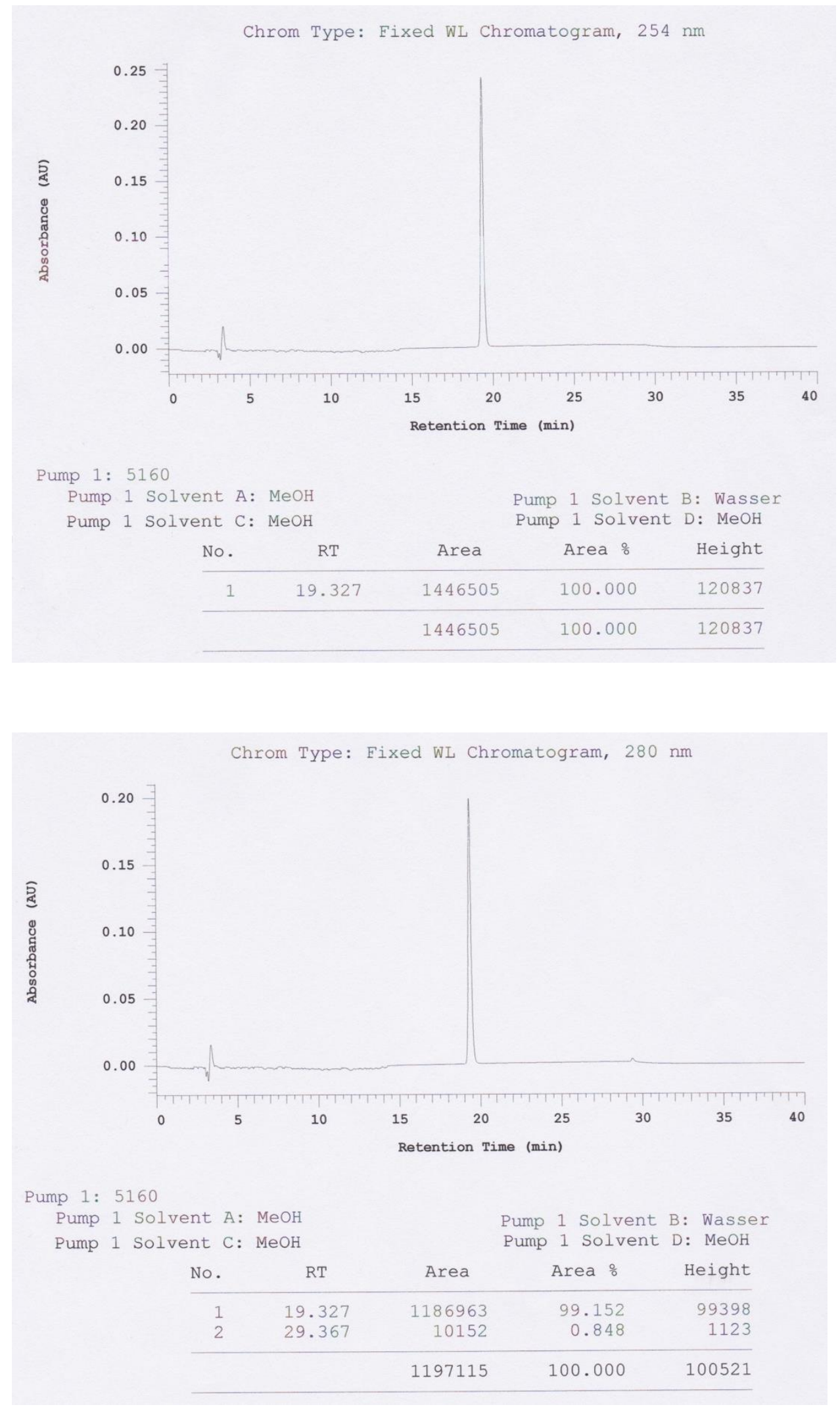
7-Methoxy-1H-indole-3-carboxylic acid methyl ester (27):
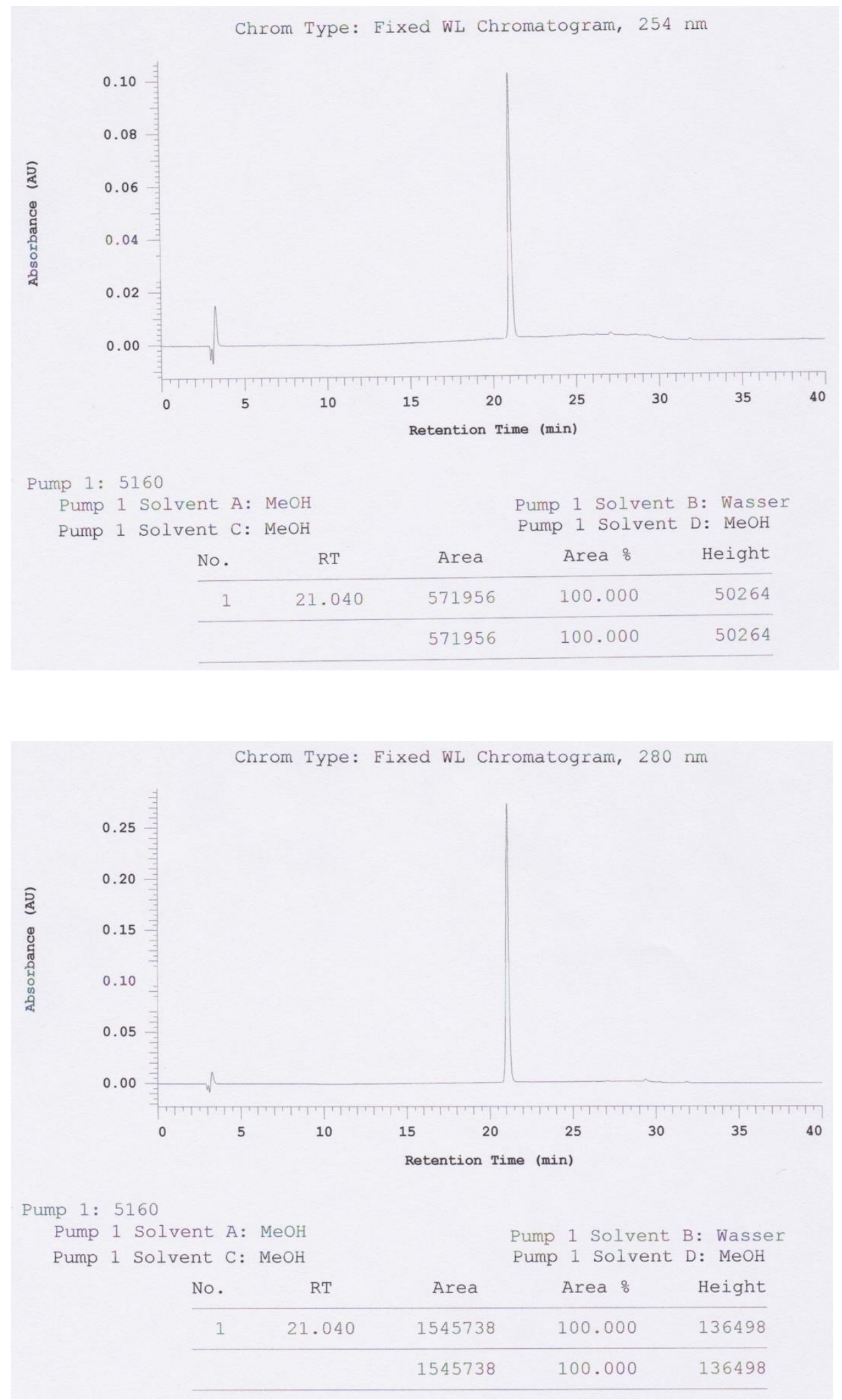
5-Bromo-1 $H$-indole-3-carboxylic acid methyl ester (28):
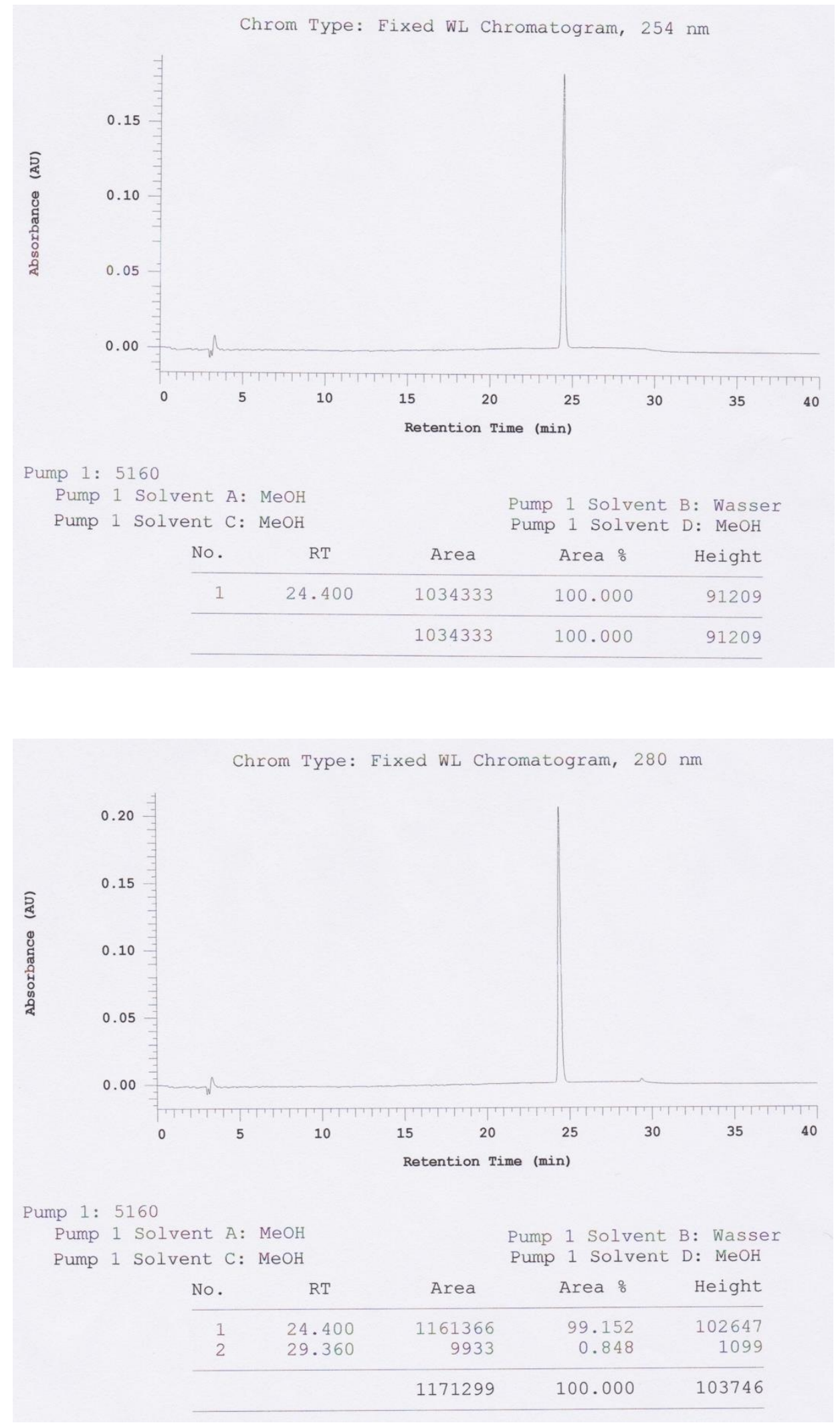


\section{5-Phenyl-1H-indole-3-carboxylic acid methyl ester (29):}

Chrom Type: Fixed WL Chromatogram, $254 \mathrm{~nm}$

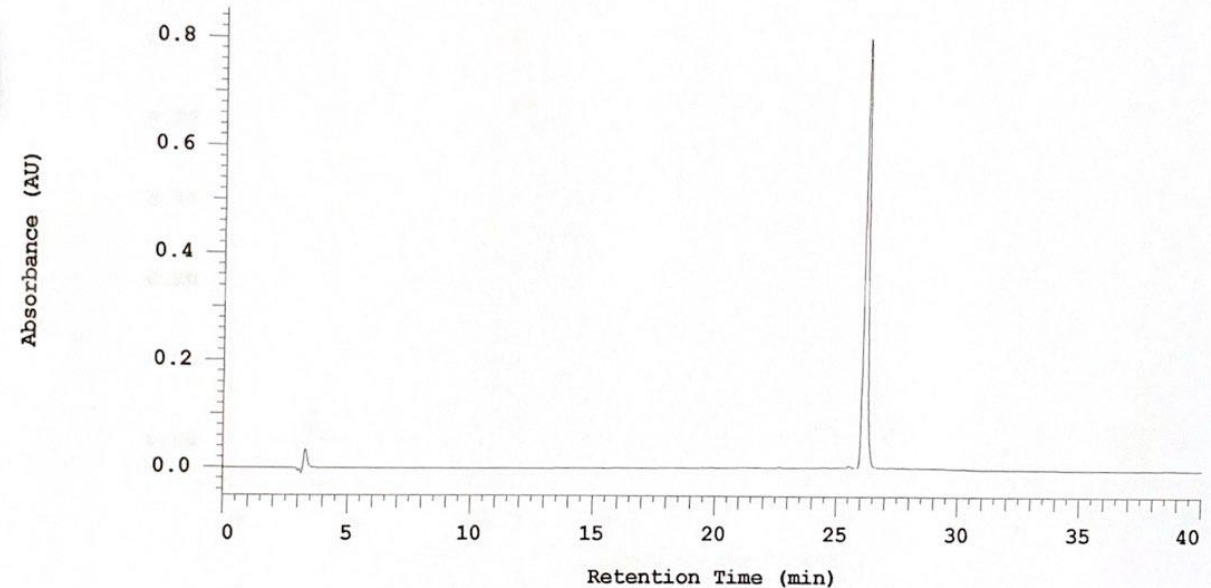

Pump 1: 5160

Pump 1 Solvent A: MeOH Pump 1 Solvent B: Wasser

Pump 1 Solvent C: $\mathrm{MeOH} \quad$ Pump 1 Solvent D: $\mathrm{MeOH}$

\begin{tabular}{rrrrr} 
No. & RT & \multicolumn{1}{c}{ Area } & Area $\%$ & Height \\
\hline 1 & 25.493 & 16369 & 0.377 & 1502 \\
2 & 26.113 & 4321096 & 99.623 & 403279 \\
\hline & & 4337465 & 100.000 & 404781
\end{tabular}

Chrom Type: Fixed WL Chromatogram, $280 \mathrm{~nm}$

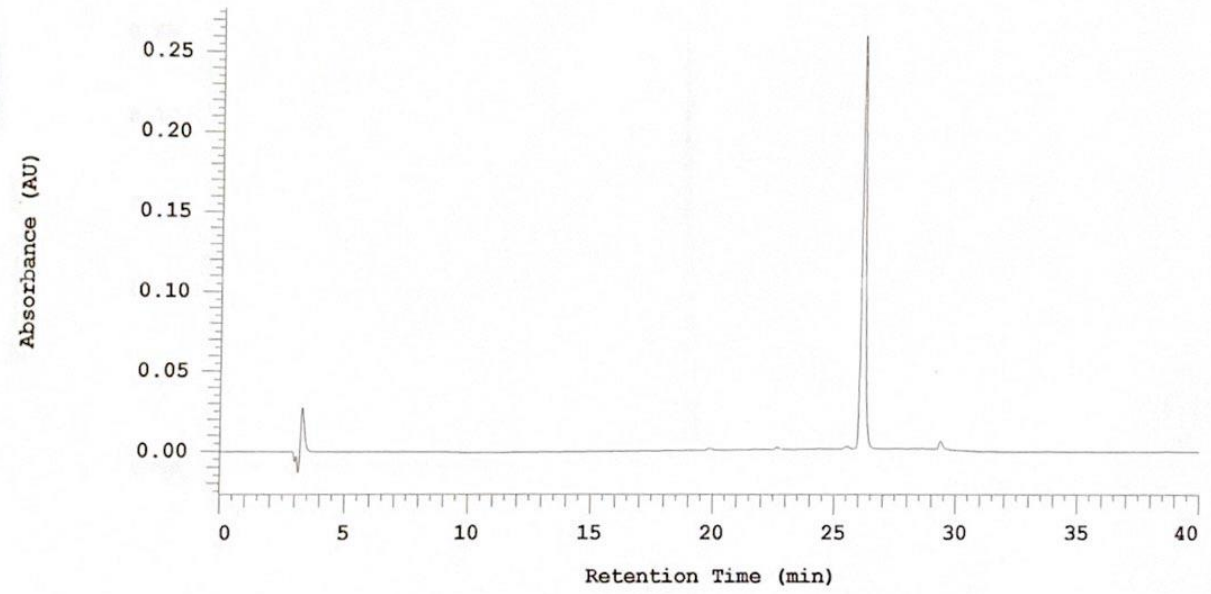

Pump 1: 5160

Pump 1 Solvent A: MeOH Pump 1 Solvent B: Wasser

Pump 1 Solvent C: $\mathrm{MeOH}$

Pump 1 Solvent D: $\mathrm{MeOH}$

\begin{tabular}{rrrrr} 
No. & \multicolumn{1}{c}{ RT } & \multicolumn{1}{c}{ Area } & Area $\%$ & Height \\
\hline 1 & 22.647 & 5437 & 0.390 & 580 \\
2 & 25.493 & 5605 & 0.402 & 663 \\
3 & 26.113 & 1383479 & 99.208 & 130264 \\
\hline & & 1394521 & 100.000 & 131507
\end{tabular}


5-Benzyl-1H-indole-3-carboxylic acid methyl ester (30):
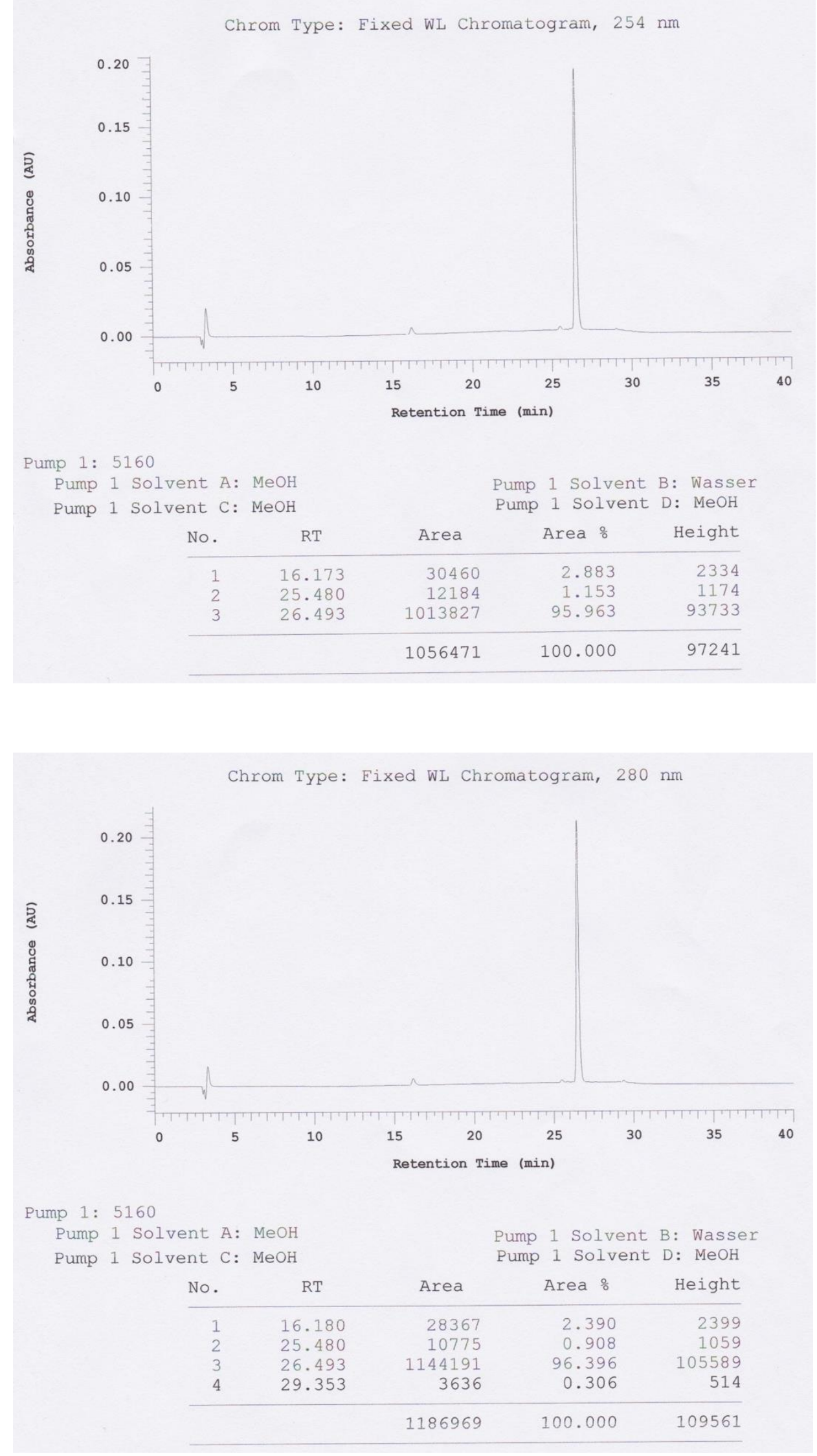
5-Phenyl-1-methylindole-3-carboxylic acid methyl ester (31):

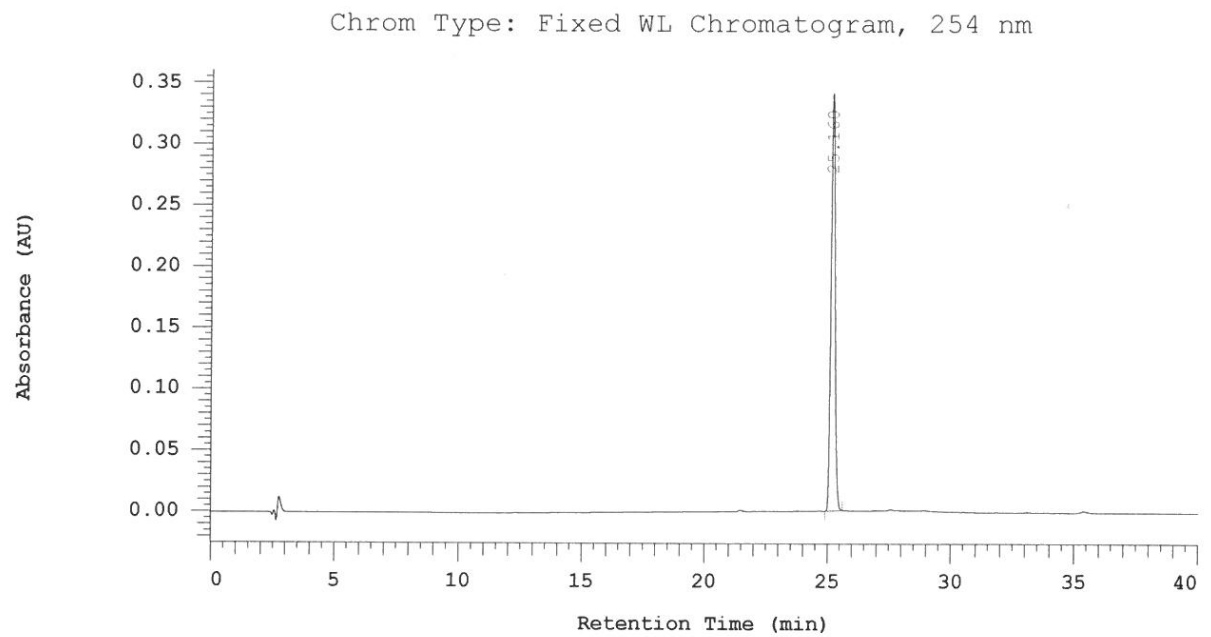

Pump 1: 5160

Pump 1 Solvent A: $\mathrm{MeOH}$

Pump 1 Solvent $\mathrm{C}$ : $\mathrm{MeOH}$

Pump 1 Solvent B: Wasser

Pump 1 Solvent D: $\mathrm{MeOH}$

\begin{tabular}{ccccc} 
No. & RT & Area & Area & Height \\
\hline 1 & 25.160 & 1919342 & 100.000 & 170134 \\
\hline & 1919342 & 100.000 & 170134 \\
\hline
\end{tabular}

Chrom Type: Fixed WL Chromatogram, $280 \mathrm{~nm}$

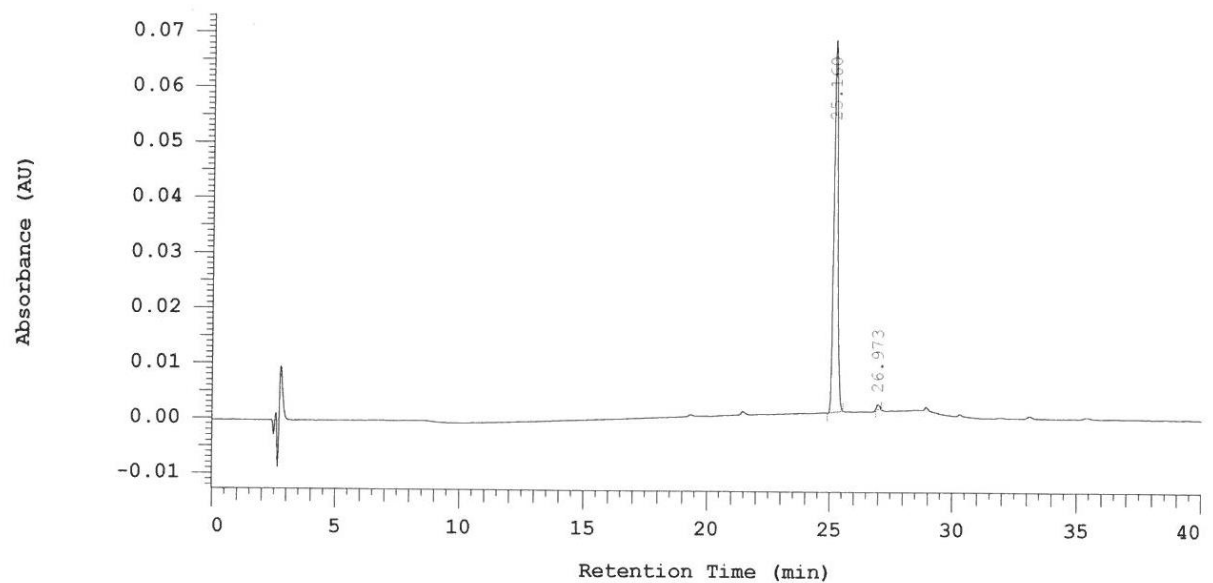

Pump 1: 5160

Pump 1 Solvent A: $\mathrm{MeOH}$

Pump 1 Solvent $\mathrm{C}: \mathrm{MeOH}$

Pump 1 Solvent B: Wasser

Pump 1 Solvent D: $\mathrm{MeOH}$

\begin{tabular}{rrrrr} 
No. & RT & Area & Area & Height \\
\hline 1 & 25.160 & 378207 & 99.104 & 33665 \\
2 & 26.973 & 3418 & 0.896 & 428 \\
\hline & 381625 & 100.000 & 34093 \\
\hline
\end{tabular}




\section{$N$-methyl-1-methyl-5-phenylindole-3-carboxamide (32):}
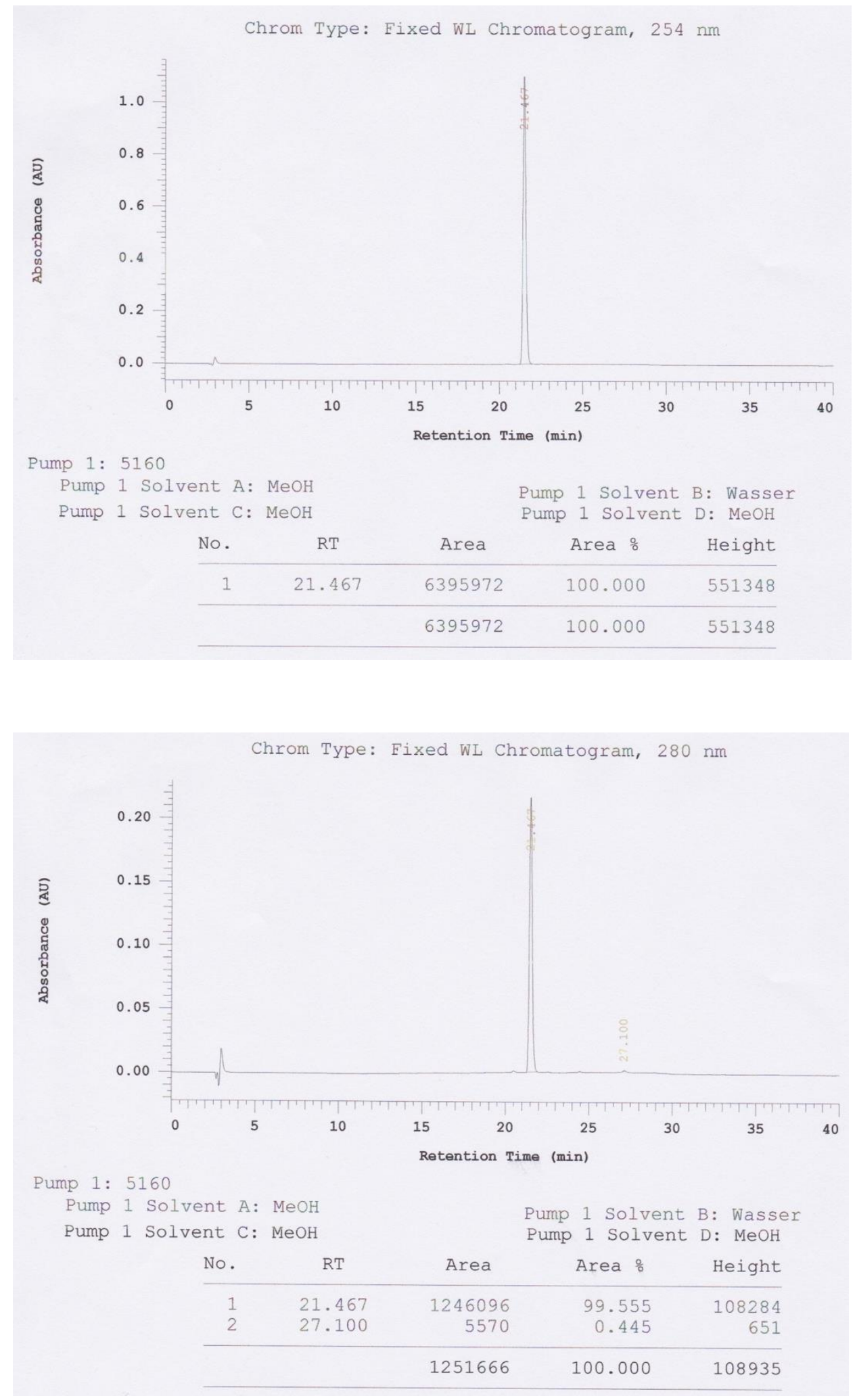


\section{$\mathrm{N}$-ethyl-1-methyl-5-phenylindole-3-carboxamide (33):}
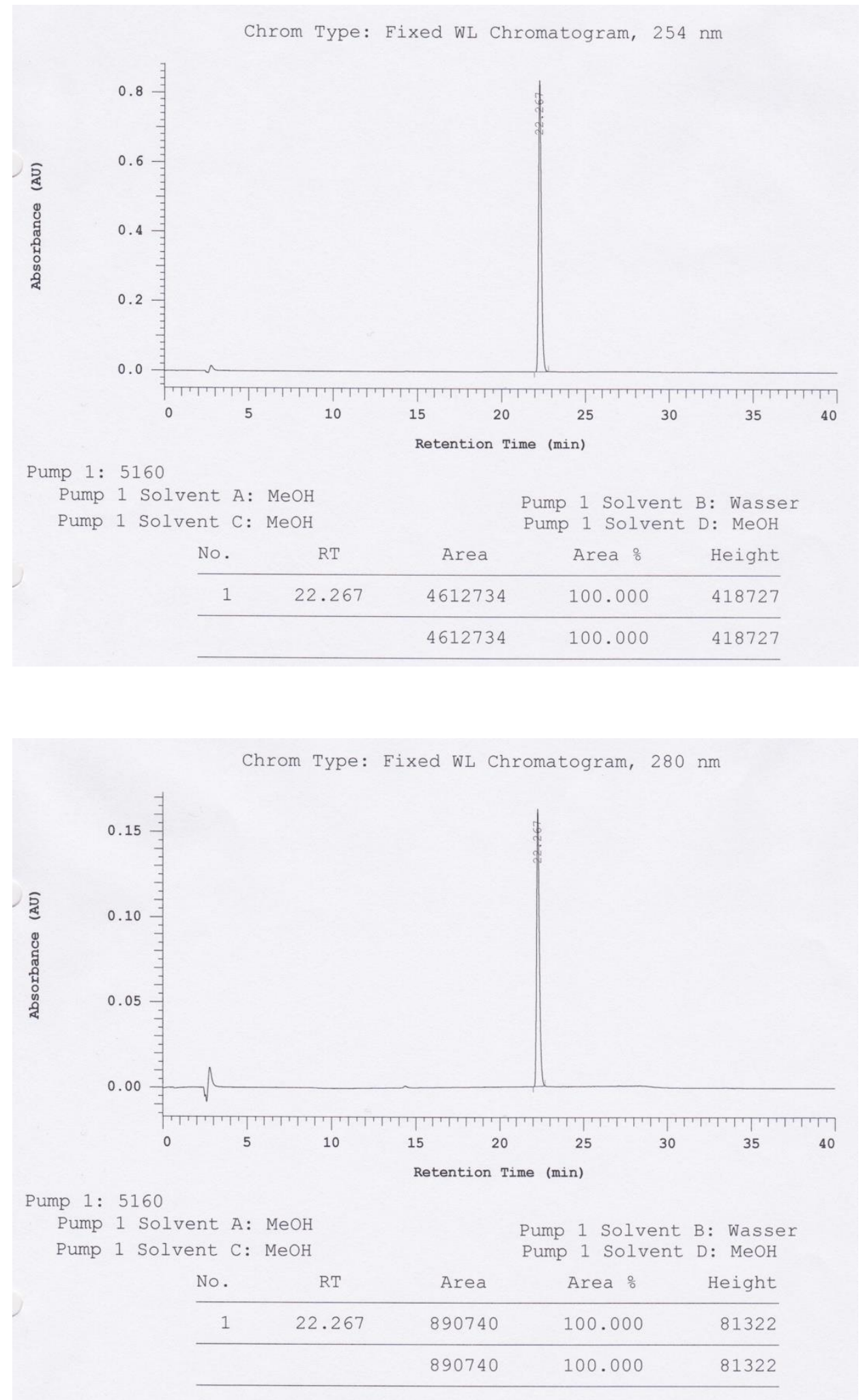


\section{$\mathrm{N}$-dimethyl-1-methyl-5-phenylindole-3-carboxamide (34):}
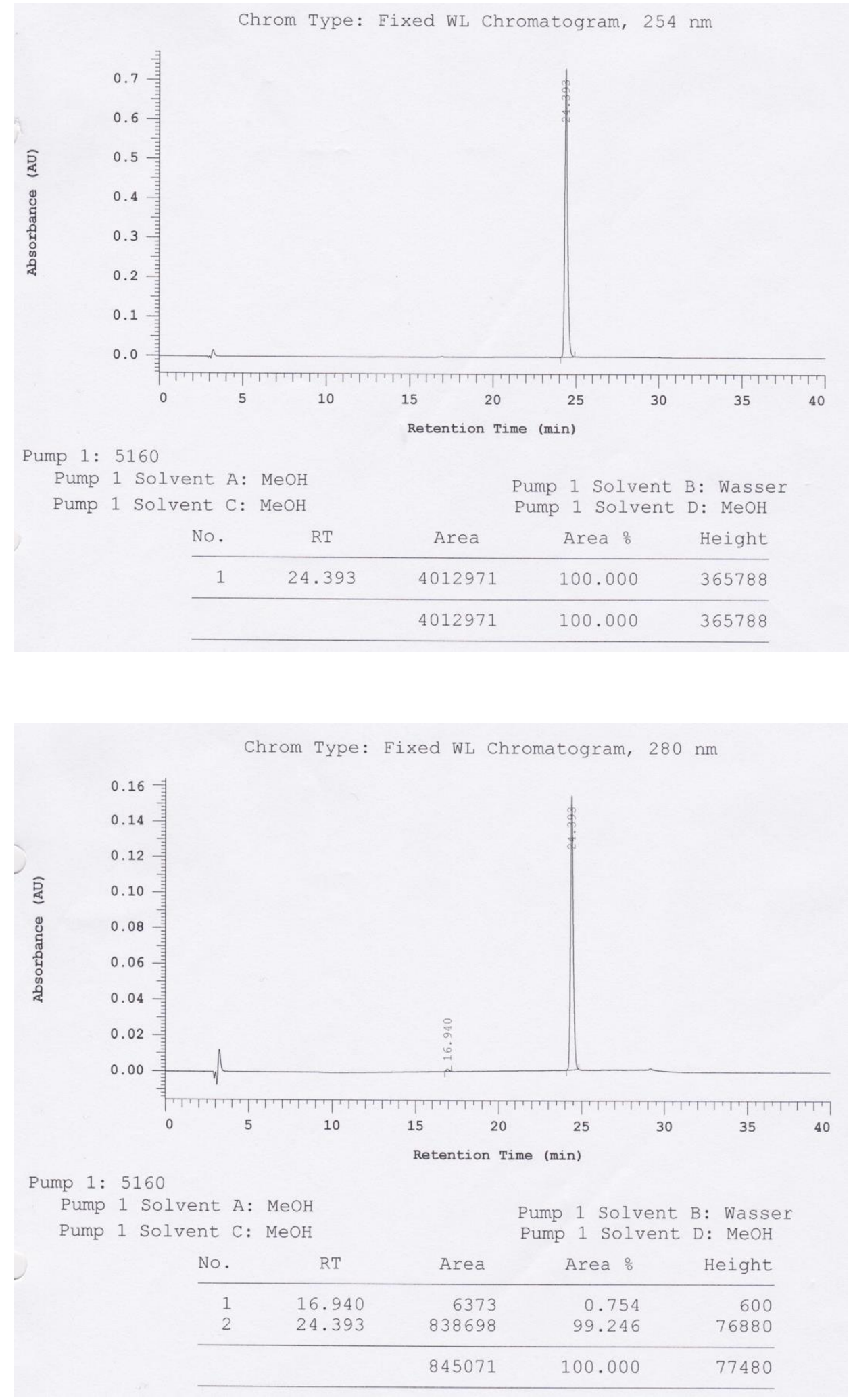
5-(Furan-2-yl)-1-methylindole-3-carboxylic acid methyl ester (35):
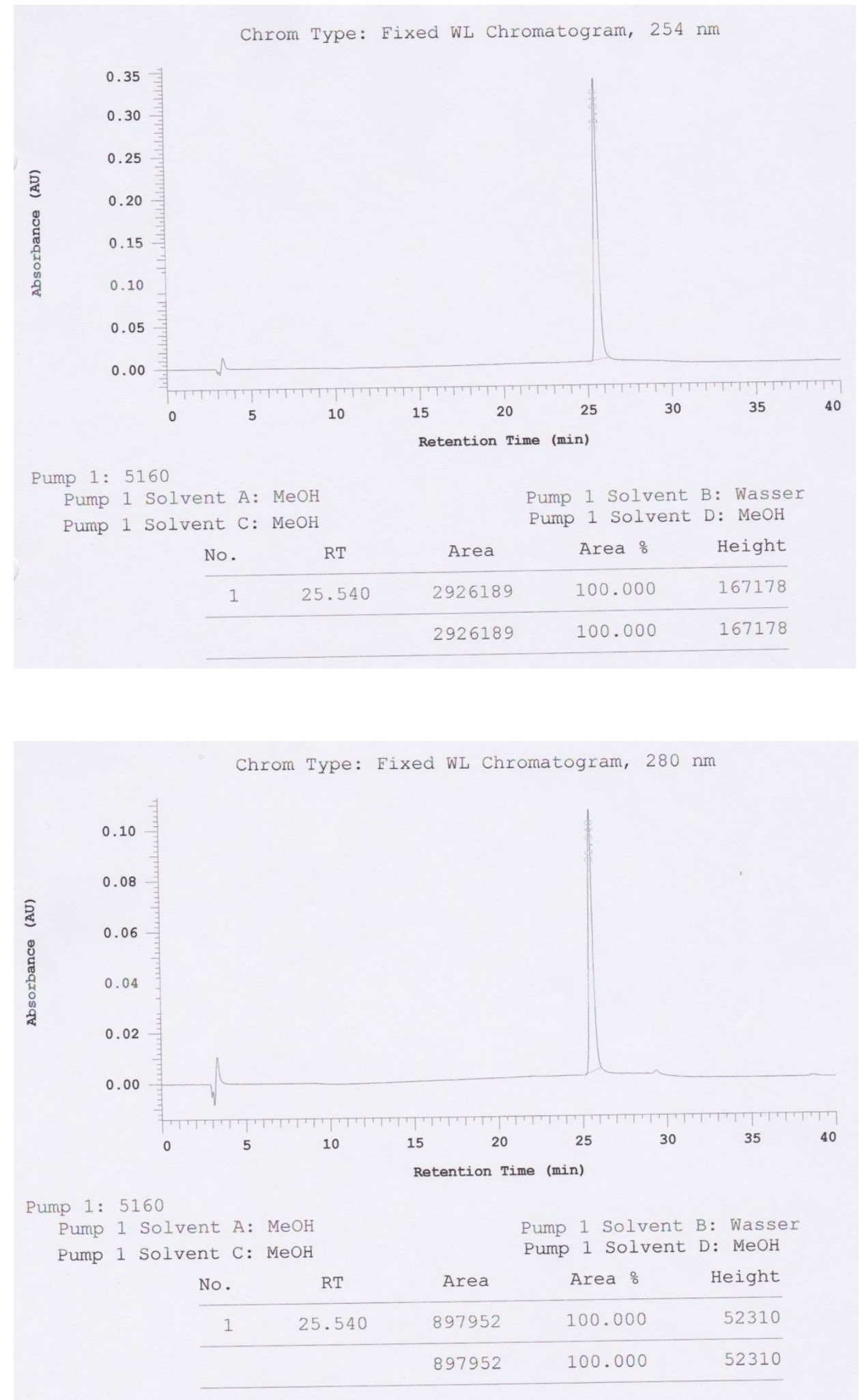
5-(Furan-3-yl)-1-methylindole-3-carboxylic acid methyl ester (36):
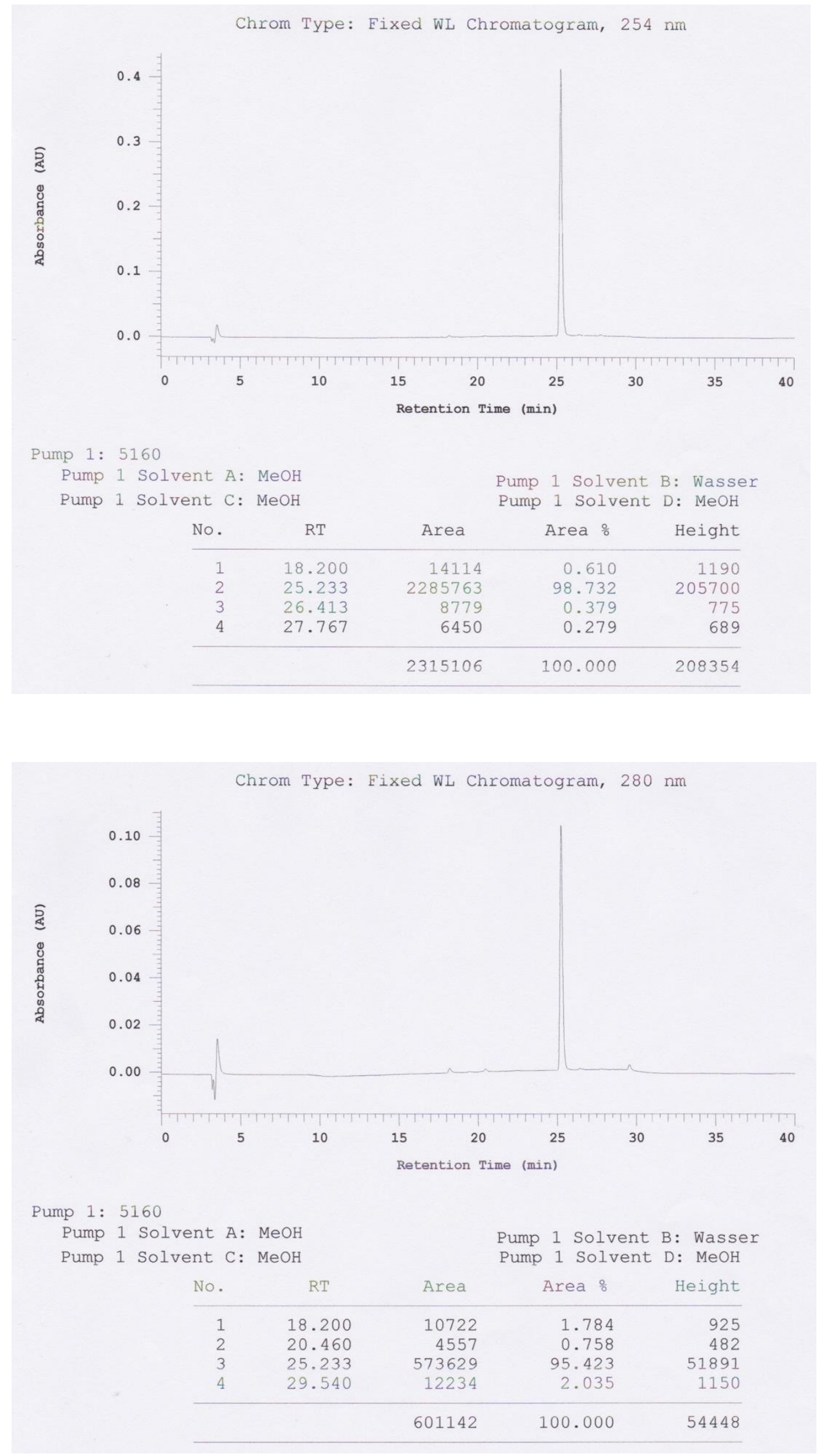
5-(Thiophen-2-yl)-1-methylindole-3-carboxylic acid methyl ester (37):
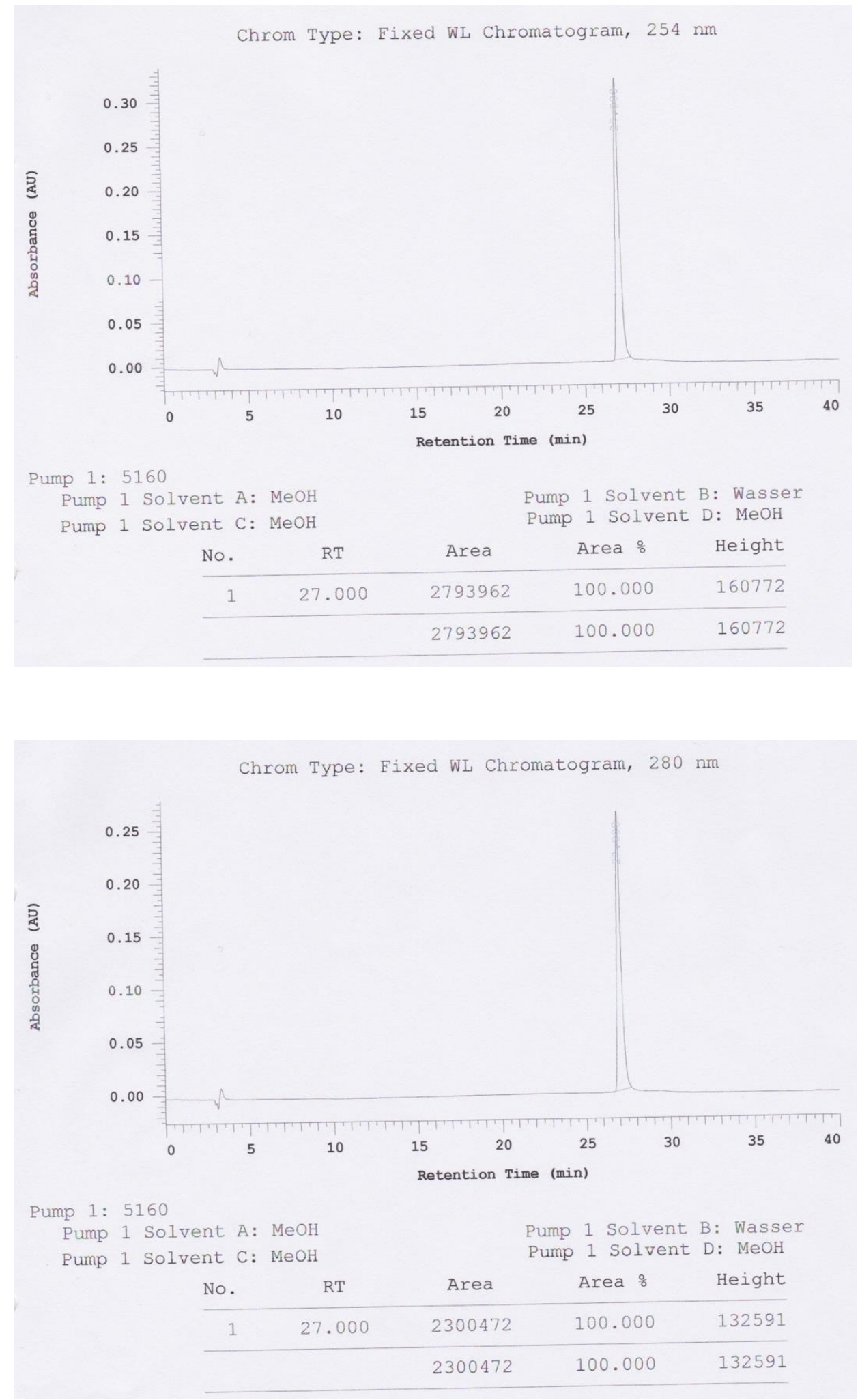
5-(Thiophen-3-yl)-1-methylindole-3-carboxylic acid methyl ester (38):

Chrom Type: Fixed WL Chromatogram, 254 nm

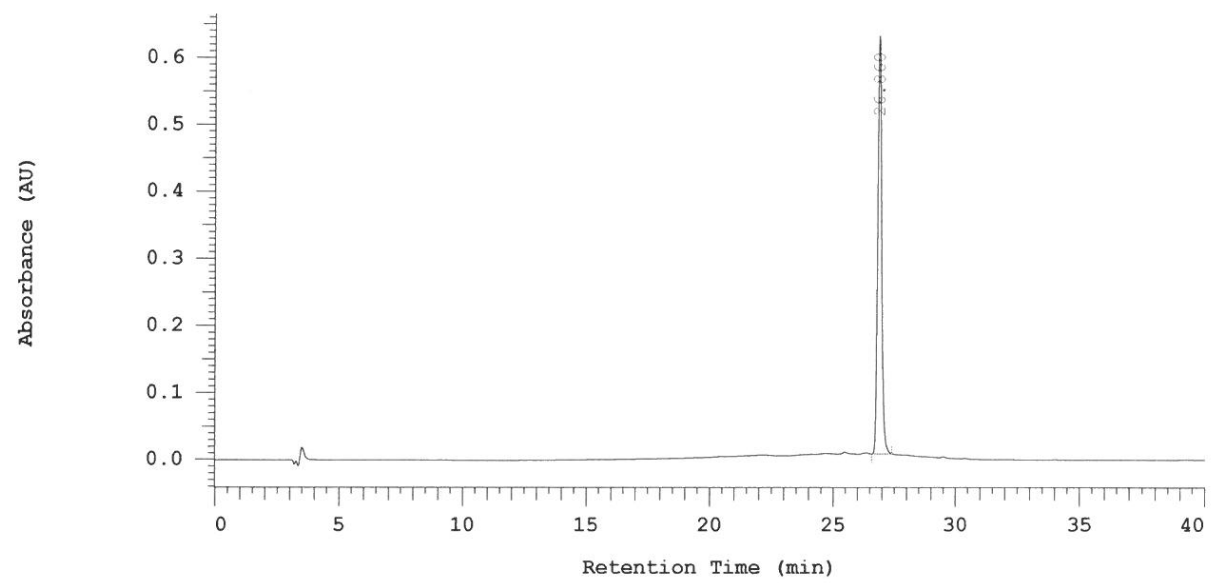

Pump 1: 5160

Pump 1 Solvent A: $\mathrm{MeOH} \quad$ Pump 1 Solvent B: Wasser

Pump 1 Solvent $\mathrm{C}$ : $\mathrm{MeOH} \quad$ Pump 1 Solvent D: MeOH

\begin{tabular}{ccccc} 
No. & RT & Area & Area & Height \\
\hline 1 & 26.860 & 3446143 & 100.000 & 311366 \\
\hline & 3446143 & 100.000 & 311366
\end{tabular}

Chrom Type: Fixed WL Chromatogram, $280 \mathrm{~nm}$

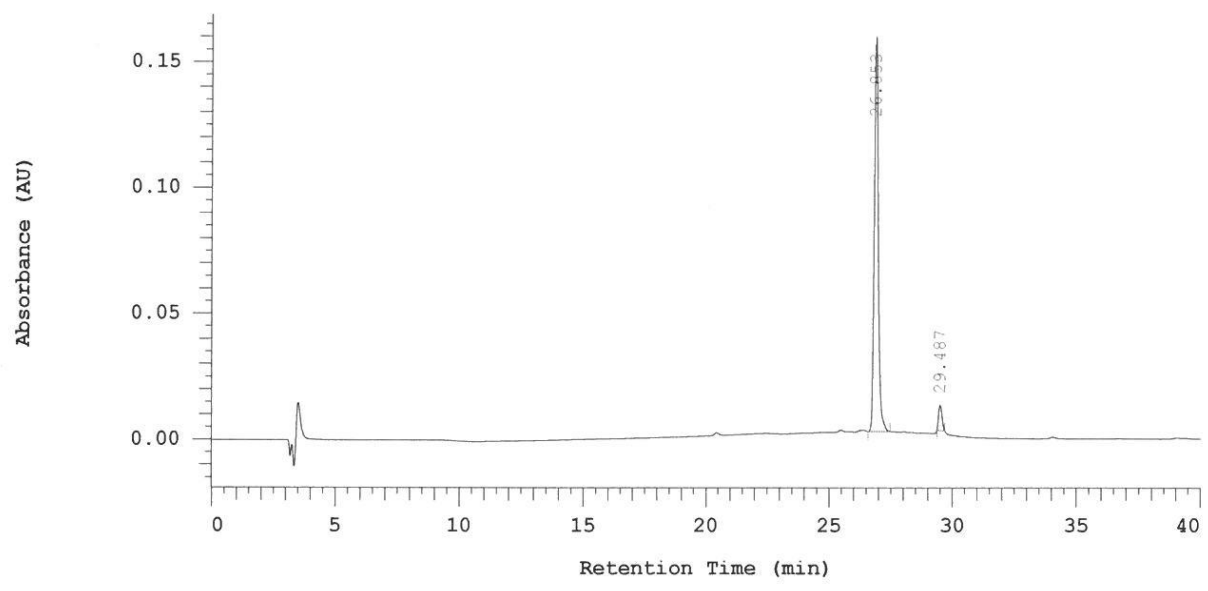

Pump 1: 5160

Pump 1 Solvent A: $\mathrm{MeOH} \quad$ Pump 1 solvent B: Wasser

Pump 1 Solvent $\mathrm{C}$ : $\mathrm{MeOH} \quad$ Pump 1 Solvent D: MeOH

\begin{tabular}{rrrrr} 
No. & RT & Area & Area & Height \\
\hline 1 & 26.853 & 877309 & 95.069 & 78358 \\
2 & 29.487 & 45503 & 4.931 & 4979 \\
\hline & & 922812 & 100.000 & 83337
\end{tabular}


5-(Pyridin-4-yl)-1-methylindole-3-carboxylic acid methyl ester (39):
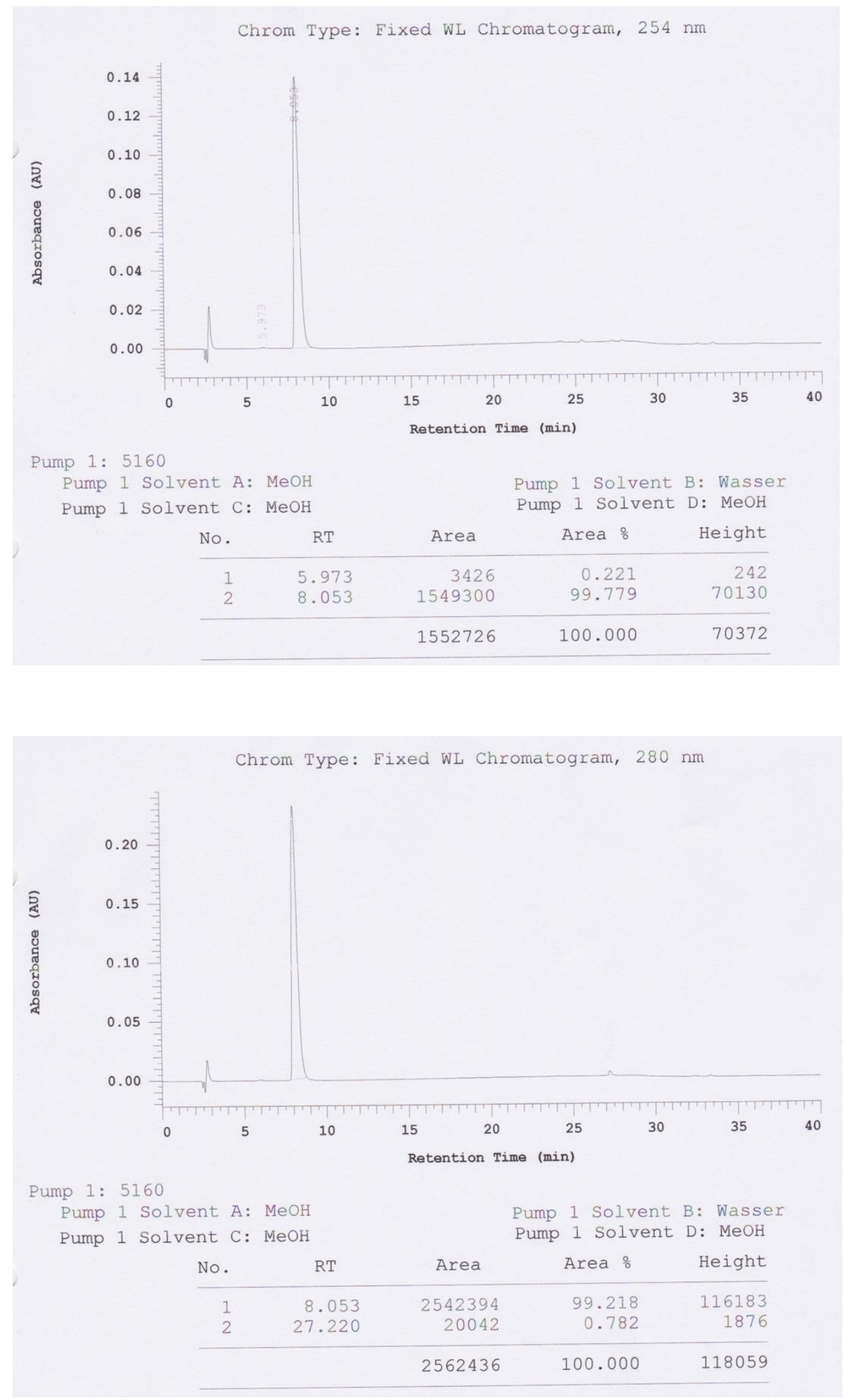
5-(Pyridin-3-yl)-1-methylindole-3-carboxylic acid methyl ester (40):
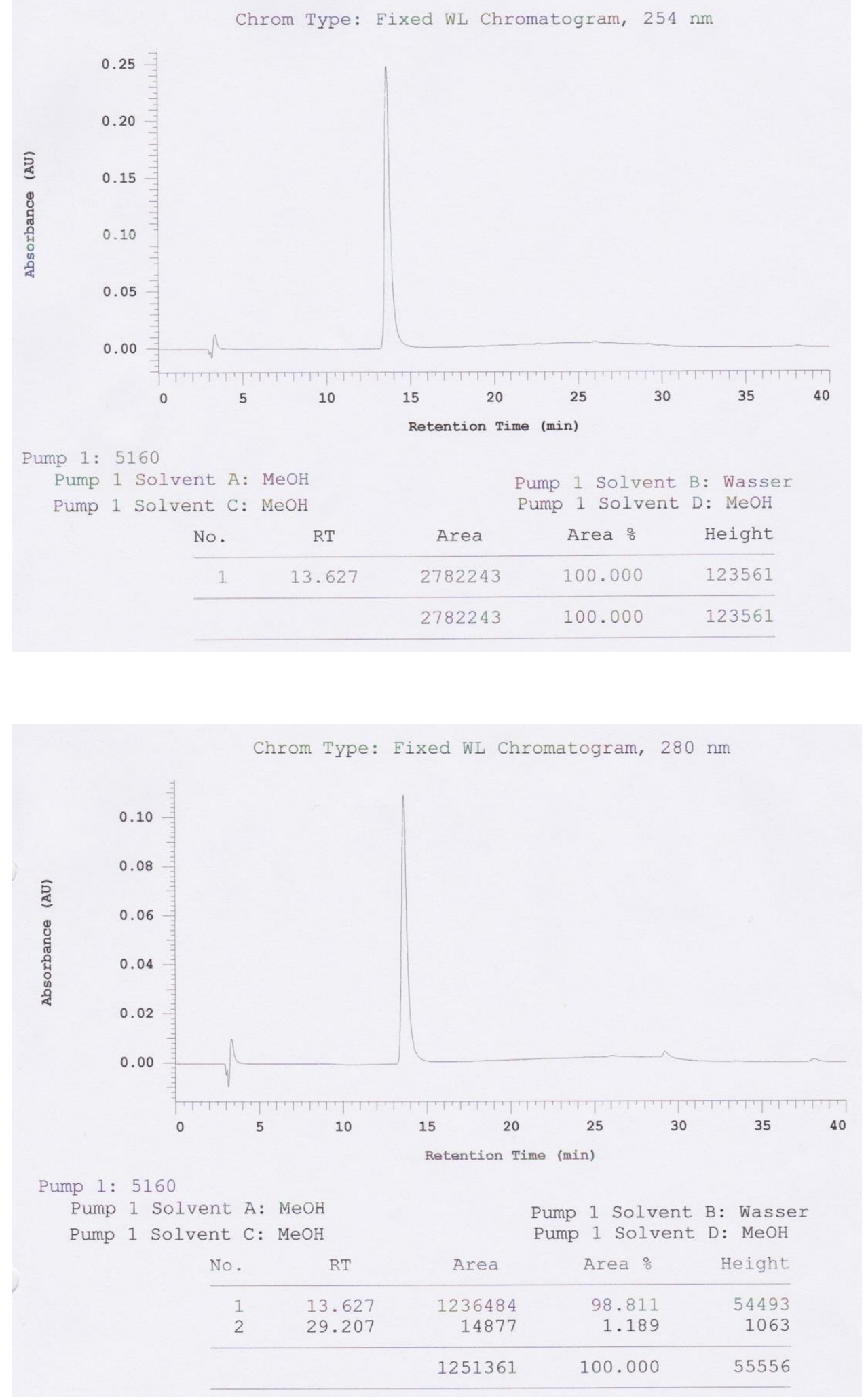
7-Chloro-1-methyl-5(pyridin-3-yl)-1H-indole-3-carboxylic acid methyl ester (41):
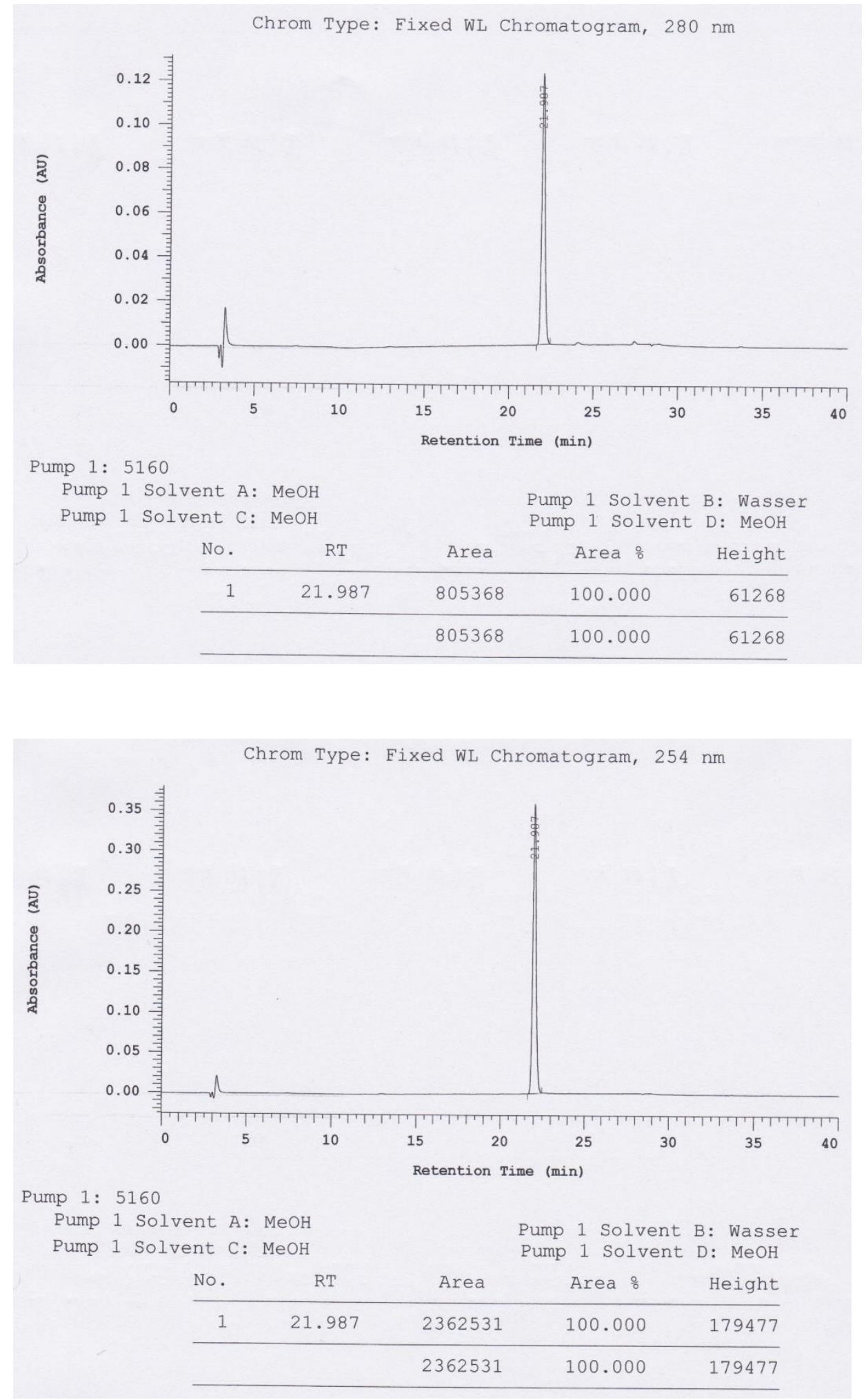


\section{5-(Pyridin-3-yl)-1H-indole-3-carboxylic acid methyl ester (42):}

Chrom Type: Fixed WL Chromatogram, $254 \mathrm{~nm}$

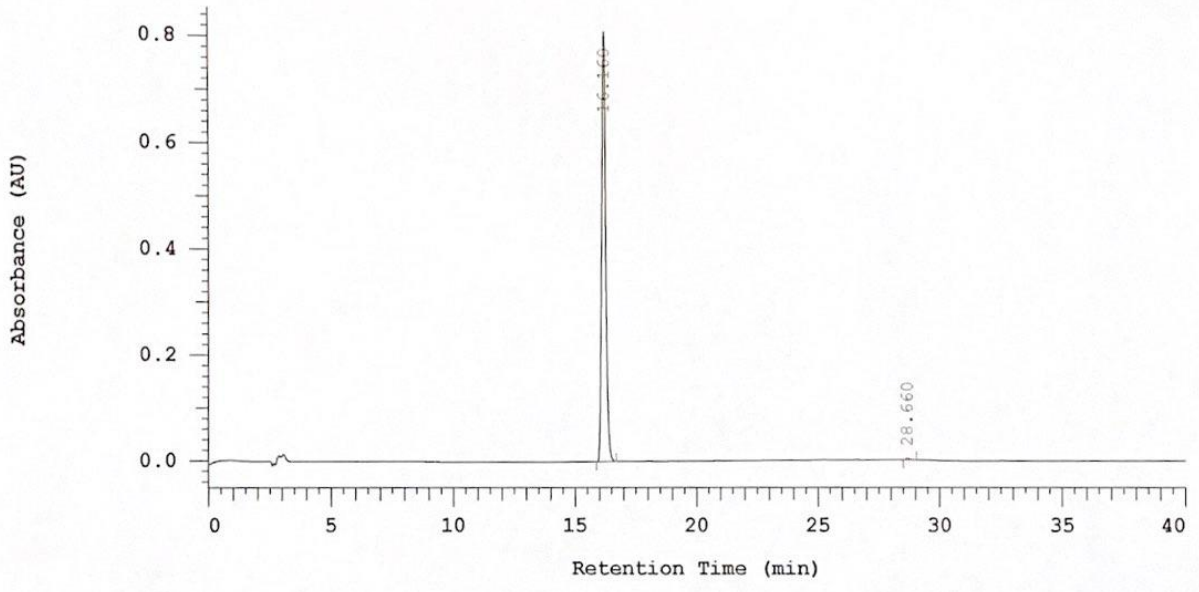

Pump 1: 5160

Pump 1 Solvent A: $\mathrm{MeOH} \quad$ Pump 1 Solvent B: Wasser

Pump 1 Solvent C: $\mathrm{MeOH} \quad$ Pump 1 Solvent D: $\mathrm{MeOH}$

\begin{tabular}{rrrrr} 
No. & RT & \multicolumn{1}{c}{ Area } & Area \% & Height \\
\hline 1 & 16.160 & 4536785 & 99.705 & 406272 \\
2 & 28.660 & 13424 & 0.295 & 1316 \\
\hline & & 4550209 & 100.000 & 407588
\end{tabular}

Peak rejection level: 0

Chrom Type: Fixed WL Chromatogram, $280 \mathrm{~nm}$

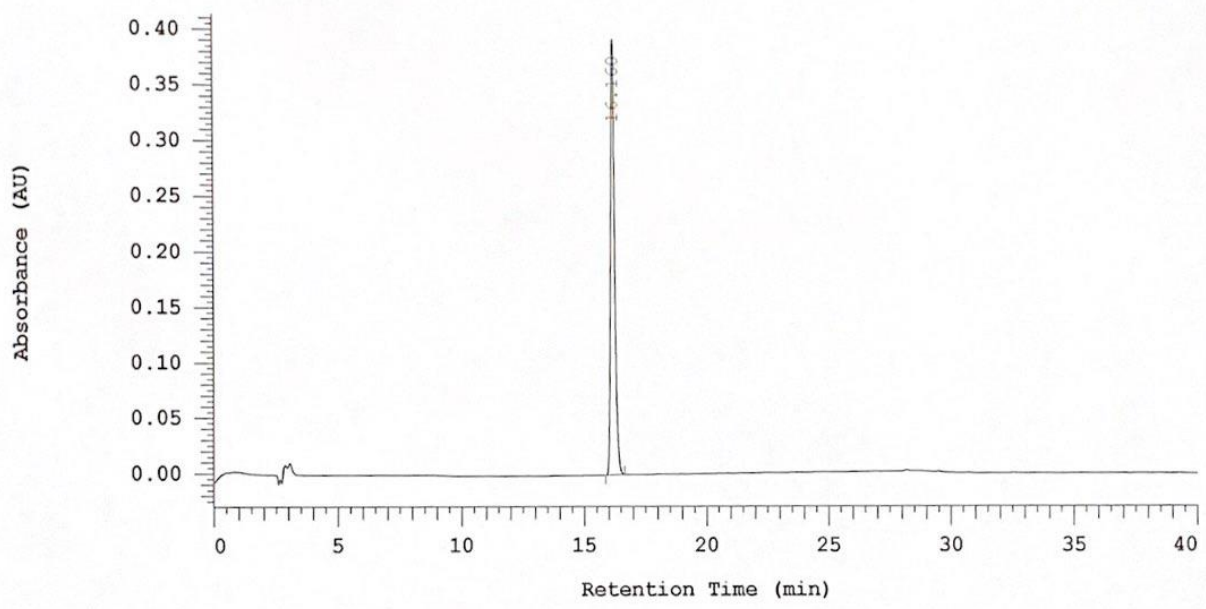

Pump 1: 5160

Pump 1 Solvent A: $\mathrm{MeOH}$

Pump 1 Solvent B: Wasser

Pump 1 Solvent C: $\mathrm{MeOH}$

Pump 1 Solvent D: $\mathrm{MeOH}$

\begin{tabular}{ccccc} 
No. & RT & Area & Area $\%$ & Height \\
\hline 1 & 16.160 & 2198212 & 100.000 & 197051 \\
\hline & & 2198212 & 100.000 & 197051
\end{tabular}

Peak rejection level: 0 